\title{
Modelling the vibrationally mediated photo-dissociation of acetylene.
} $\dagger$

\author{
Christopher Robertson ${ }^{a, b}$ and Graham A. Worth ${ }^{a, c}$
}

\author{
Received Xth $X X X X X X X X X X 20 X X$, Accepted Xth $X X X X X X X X X 20 X X$ \\ First published on the web Xth $X X X X X X X X X X 200 X$ \\ DOI: $10.1039 / b 000000 x$
}

\begin{abstract}
A ten singlet state vibronic coupling Hamiltonian was constructed describing the seven internal coordinates of acetylene. A $\Sigma_{g}^{+}$symmetry-adapted polynomial expansion of the nuclear coordinates over diabatic elements was used to fit adiabatic energies obtained from ab-initio calculations. The fitted vibronic Hamiltonian was subsequently used to model vibrationally mediated photo-dissociation (VMD) experiments. The model suggests that some control over dissociation channels might be achieved by choosing different ranges of pre-excitation and UV-excitation energies.
\end{abstract}

\section{Introduction}

Acetylene represents the simplest molecule that contains all the features of vibrational dynamics of polyatomic systems ${ }^{1}$ and has labile $\mathrm{H}$ atoms that undergo large amplitude motions. It was the first molecule to be shown to have differing equilibrium structures in the excited and ground states (trans and linear, respectively) ${ }^{2,3}$. Its photo fragment, the $\mathrm{C}_{2} \mathrm{H}$ radical, was observed in the interstellar medium ${ }^{4}$ and is a reactive intermediate in combustion processes ${ }^{5}$. To understand the details of the photo-excited dynamics of molecules such as this, quantum dynamics simulations can provide useful information by describing the non-adiabatic reactive pathways accessible after the absorption of a photon ${ }^{6}$. To do this we need to construct a model which adequately describes the excited state landscape of acetylene.

A standard approach for these problems is to construct a vibronic coupling $\operatorname{model}^{7}$ : a simple polynomial expansion of diabatic potential energy surfaces and couplings. The expansion coefficients are chosen so that the eigenvalues of the potential operator map on to the adiabatic potential surfaces. Usually normal mode coordinates are used and the surfaces are expanded around the Frank-Condon point. Such models have been shown to reproduce the short-time dynamics of non-adiabatic systems ${ }^{8}$. We have constructed a vibronic model which describes the femtosecond dynamics of acetylene in the first three singlet adiabatic excited states, including non-adiabatic effects between them as Renner-Teller (RT) and

$\dagger$ Electronic Supplementary Information (ESI) available: See DOI: 10.1039/b000000x/

${ }^{a}$, The University of Birmingham, Edgbaston, Birmingham, B15 2TT, UK

${ }^{b}$, Present Address: University of Warwick., Gibbet Hill, Coventry, CV4 7AL, UK ; E-mail: C.Robertson@warwick.ac.uk

${ }^{c}$, Present Address: University College London, 20 Gower St., London, WC1H OAJ, UK ; E-mail: g.a.worth@ucl.ac.uk pseudo Jahn-Teller (pJT) diabatic coupling. We used a set of curvilinear coordinates which describe trans and cis displacements. These form $\Pi$ Irreducible representations (IrReps) for which $\Sigma^{+}$symmetry adapted functions can be used to construct model diabatic potential. Details on the generation of such a basis are given in a previous paper ${ }^{9}$. In that same paper, the absorption spectra for the validation of the model containing only the $\Pi$ coordinates was presented. This required 10 -states in the model to explicitly represent any pJT and RT coupling the three lowest singlet exited states have to higher lying states.

In this article we have expanded the model to include dissociation coordinates and then used it to model the vibrationally mediated (photo) dissociation (VMD) of acetylene. Since the propagation of a full internal coordinate wavepacket for many tens of picoseconds and the construction of a vibronic model coupling triplet and singlet states are beyond the scope of this work, we concentrate in simulating those experiments that remain in the singlet state landscape and are expected to occur in the femtosecond regime. A number of vibrational states localised in the $\mathrm{CH}$ stretch local modes are prepared using ladder operators, followed by UV-excitation using a Gaussian enveloped sinusoidal function representing a experimental pulse polarised laser field. Dissociation cross sections are calculated for all possible angles, providing new insights into this photoprocess. The model suggests that for energies in the range 6.4$7.0 \mathrm{eV} \mathrm{S}_{1}$ can give trans or near-linear geometries depending on the IR pre-excitation of $\mathrm{CH}$ stretch local modes. Similar control can result in trans or cis geometries in the range 7-8 $\mathrm{eV}$ for state $\mathrm{S}_{2}$. 


\subsection{Singlet excited state landscape}

The absorption spectrum has been recorded in the range 5.4$7.8 \mathrm{eV}^{10}$. The maximum intensity of the $\mathrm{S}_{1} \leftarrow \mathrm{S}_{0}$ transition lies near the vertical transition $(\sim 6.9 \mathrm{eV})$, despite all valence states in this region being optically forbidden at a linear geometry. In this region we find singlet valence and Rydberg states. Acetylene's valence states appearing in its absorption spectra arise from states formed from $\pi \rightarrow \pi^{*}$ orbital excitations. From the degenerate HOMO and LUMO orbitals of symmetry $\Pi \otimes \Pi$ we can obtain $\Sigma$ and $\Delta$ ungerade triplet and singlet states. Double excitations can in turn produce similar gerade states. The contribution of a $\pi^{*}$ orbital to valence states has the effect of stabilising the molecule towards a bent geometry. In contrast, Rydberg states found in this range arise from orbital excitations $\pi \rightarrow n / \Lambda$ ( $\Lambda=$ hydrogenic wavefunction $)$ which give stabilisation energies favouring a linear geometry ${ }^{11}$.

The $S_{1}$ minimum has been found experimentally and theoretically to be at trans bent geometry with an isomerisation barrier to its cis conformer via a torsional motion. The transition state geometry for cis-trans isomerisation has been calculated to be with one $\mathrm{CCH}$ angle being near-linear and the other at $120^{\circ} 12$. For $\mathrm{S}_{2}$, a non-planar cisoid (cis like) minimum geometry has been calculated using ab initio methods ${ }^{12,13}$. Table 1 provides some critical points found in the literature as well as their adiabatic transition energies.

In its ground state global minimum geometry acetylene belongs to the $\mathrm{D}_{\infty \mathrm{h}}$ point group and is subject to Renner-Teller (RT) and pseudo Jahn-Teller (pJT) stabilisation effects. $\mathrm{S}_{2 / 3}$ form a degenerate $\Delta_{u}$ state which can exhibit RT effects. Cederbaum et al were the first to carefully analyse the effect of vibronic coupling in linear molecular systems ${ }^{14}$ and their signature in spectra. More recently Bersuker and Liu ${ }^{15}$ highlighted the small contribution RT might have to the stabilisation energy of degenerate states. This study was followed by work proving that for RT systems, all instabilities arise from pJT coupling, having implications on spectroscopic selection rules, transition dipole moments and line intensities ${ }^{16}$.

Singlet valence states leading to dissociation correlate adiabatically with two doublet states; the $\tilde{X}^{2} \Sigma^{+}$ground state $(\sim$ $\left.5.71 \mathrm{eV}^{17}\right)$ and a degenerate $\tilde{A}^{2} \Pi$ state $(6.17 \mathrm{eV}$ from the singlet ground state minima using ab-initio methods ${ }^{13}$ ) (see Tab 4). The most careful theoretical study of critical points in the excited state landscape was done by Cui and Morokuma ${ }^{13,18}$, proposing a dissociation mechanism near the experimentally observed threshold. Starting from $\mathrm{S}_{1}$, they distinguished possible pathways, as a function of energy, leading to either the doublet ground state $\tilde{X}^{2} \Sigma^{+}$or excited $\tilde{A}^{2} \Pi$ states of the $\mathrm{C}_{2} \mathrm{H}$ moiety. From $S_{1}$ they found a transition state barrier at 6.48 $\mathrm{eV}$ (including ZPE) to an adiabatic dissociation leading to the $\tilde{A}^{2} \Pi$ state $(6.15 \mathrm{eV})$. An alternative route to the same doublet state but with a lower barrier of $6.28 \mathrm{eV}$ (including ZPE) to dissociation occurs via a conical intersection with $S_{2}$ at an out-of-plane geometry and then possibly via an avoided crossing at $6.10 \mathrm{eV}$. They suggest this pathway should be a slow process compared to tunnelling directly from $S_{1}$ at such energies. More recent work ${ }^{11}$ suggests another avoided crossing is found by dissociation from the linear geometry; a degenerate $\Pi_{u}$ state is strongly pre-dissociated by higher lying $\Pi_{g}$ states which then exhibit an avoided crossing with $\mathrm{S}_{0}$ at a $\mathrm{CH}$ bond length $2.5 \AA$ (discussed further in the following subsection).

The lowest energy pathway to dissociation has received abundant attention from the spectroscopy community ${ }^{17,19}$; the common view is that the $S_{1}$ state undergoes intersystem crossing to a manifold of triplet states where pre-dissociation takes place (a drastic decrease in fluorescence yield has been reported above $5.7 \mathrm{eV}^{20}$ ). This picture agrees with many $a b$ initio calculations ${ }^{13,21,22}$, which suggest that after intersystem crossing there would have to be a sequential energy transfer along several modes involving large molecular contortions that would make such a mechanism slow ${ }^{13}$. This agrees with the observed $\mu$ s metastable state lifetimes of the excited acetylene upon irradiation ${ }^{23,24}$ and a lower limit for a predissociation lifetime has been suggested of at least tens of picoseconds ${ }^{25}$.

\subsection{Vibrationally mediated dissociation of acetylene}

A number of experiments have probed the stability of acetylene near the adiabatic dissociation threshold energy range, suggesting singlet dissociation channels open up above $\sim 6.2$ $\mathrm{eV}$, in agreement with theory. Most of these experiments are termed vibrationally mediated (photo) dissociation (VMD) experiments and represent some of the earliest examples of control in photochemistry ${ }^{26}$; experimentalists prepare the initial state by rovibrational excitations $(\sim 1.5-2.0 \mathrm{eV})$ prior to photodissociation with UV pulses of 230-260 nm (4.7-5.4 $\mathrm{eV})$. The rovibrationally excited wavefunction correlates differently with the excited electronic states and provides new insights into the excited state topology as well as facilitating the assignment of vibrational progressions.

In the context of organic molecules, valence $\pi \pi^{*}$ orbital excitations typically do not significantly affect the $\mathrm{CH}$ bond length, consequently vibronic $\mathrm{CH}$ stretch bands are usually missing from electronic transitions. VMD allows experimentalists to probe the excited state surface along these modes as well as to drive photochemical reactions (especially true due to the high IR absorption coefficients for these modes) ${ }^{20}$. These changes in Frank-Condon factors effectively influence the electronic excitation.

For acetylene, excitation at trans bent geometries is experimentally observed to have better transition probabilities, which is theoretically supported by the strong vibronic dependence on the transition dipole moment surfaces (shown below 


\begin{tabular}{|c|c|c|c|}
\hline Geometry & Reference & $\begin{array}{c}\text { Internal Coordinates } \\
\mathrm{CH} / \mathrm{CC} / \mathrm{Ang} / \mathrm{Dih}\end{array}$ & $\begin{array}{l}\text { Adiab. } \\
\text { ex. En. } \\
(\mathrm{eV})\end{array}$ \\
\hline \multicolumn{4}{|c|}{$\overline{\mathbf{S}_{1}}$} \\
\hline \multirow[t]{4}{*}{ trans } & Cui 97 & 1.105/1.377/122.4/NA & 5.26 \\
\hline & Malsch 01 & $1.094 / 1.382 / 122.0 / \mathrm{NA}$ & 5.14 \\
\hline & Ventura 03 & 1.092/1.372/122.2/NA & 5.23 \\
\hline & this work & $1.105 / 1.422 / 125.3 / \mathrm{NA}$ & 5.39 \\
\hline d-trans & this work & $\begin{array}{r}(0.979 \& 1.859) / 1.362 \\
/(123.2 \& 124.7) / \mathrm{NA}\end{array}$ & 6.50 \\
\hline \multirow[t]{4}{*}{ cis } & Cui 97 & $1.107 / 1.341 /-/ \mathrm{NA}$ & 5.77 \\
\hline & Malsch 01 & 1.097/1.353/132.0/NA & 5.55 \\
\hline & Ventura 03 & 1.094/1.342/132.7/NA & 5.58 \\
\hline & this work & 1.196/1.316/129.1/NA & 5.60 \\
\hline $\mathrm{d}-c i s$ & this work & $\begin{array}{r}(1.095 \& 1.889) / 1.333 \\
/(134.2 \& 123.0) / \mathrm{NA}\end{array}$ & 6.65 \\
\hline \multirow{2}{*}{$\begin{array}{l}\text { linear } \\
\text { linear }\end{array}$} & Malsch 01 & $1.067 / 1.341 / \mathrm{NA} / \mathrm{NA}$ & 6.96 \\
\hline & this work & $1.0505 / 1.239 / \mathrm{NA} / \mathrm{NA}$ & 6.78 \\
\hline \multicolumn{4}{|c|}{$\mathbf{S}_{2}$} \\
\hline \multirow[t]{2}{*}{ trans } & Malsch 01 & 1.092/1.350/146.0/NA & 6.68 \\
\hline & Ventura 03 & 1.071/1.327/149.9/NA & 6.80 \\
\hline \multirow[t]{2}{*}{ cis } & Malsch 01 & $1.127 / 1.346 / 146.0 / \mathrm{NA}$ & 6.60 \\
\hline & Ventura 03 & 1.098/1.328/140.0/NA & 6.55 \\
\hline \multirow[t]{2}{*}{ cisoid } & this work & $\begin{array}{r}(1.216 \& 1.154) / 1.372 \\
/(137.6 \& 115.5) / 60.0\end{array}$ & 6.50 \\
\hline & this work & $\begin{array}{l}(1.116 \& 1.092) / 1.418 \\
/(116.1 \& 129.0) / 82.2\end{array}$ & 6.70 \\
\hline d-cisoid & this work & $\begin{array}{c}(1.052 \& 1.856) / 1.278 \\
/(155.1 \& 121.0) / 55.3\end{array}$ & 7.55 \\
\hline $\begin{array}{r}\mathrm{d}-\text { out of } \\
\text { plane }\end{array}$ & this work & $\begin{array}{l}(1.077 \& 1.830) / 1.311 \\
/(115.1 \& 125.0) / 105.3\end{array}$ & 7.55 \\
\hline \multirow[t]{2}{*}{ linear } & Malsch 01 & $1.061 / 1.322 / \mathrm{NA} / \mathrm{NA}$ & 7.30 \\
\hline & this work & $1.056 / 1.239 / \mathrm{NA} / \mathrm{NA}$ & 7.23 \\
\hline \multicolumn{4}{|c|}{$\mathbf{S}_{3}$} \\
\hline trans & Malsch 01 & 1.078/1.325/168.1/NA & 6.83 \\
\hline \multirow{2}{*}{$\begin{array}{c}\text { cis } \\
\text { cisoid }\end{array}$} & Malsch 01 & $1.080 / 1.328 / 169.0 / \mathrm{NA}$ & 6.82 \\
\hline & & $\begin{array}{c}(1.083 \& 1.096) / 1.310 / \\
(132.01 \& 133.4) / 76.8\end{array}$ & 6.90 \\
\hline $\begin{array}{l}\text { out of } \\
\text { plane }\end{array}$ & this work & $\begin{array}{c}(1.097 \& 1.103) / 1.377 / \\
(121.8 \& 121.8) / 96.33\end{array}$ & 6.9 \\
\hline linear & this work & $1.075 / 1.239 / \mathrm{NA} / \mathrm{NA}$ & 7.20 \\
\hline
\end{tabular}

Table 1 Acetylene excited state critical point geometries and energies for states $S_{1}-S_{3}$. If two values are given by $\&$ it means the $\mathrm{CH}$ bonds/angles where different for the two protons. Prefix drefers to a proton being stretched to near dissociation before minimising model energy. Methods: Cui ${ }^{13}$ : EOM-CCSD/cc-pVTZ (CASPT2 for energy); Malsh ${ }^{12}$ : CASSCF/ANO (PT2 for energies) ; Ventura $^{22}$ : MR-AQCC/cc-pVQZ ; this work: EOM-CCSD/aug-cc-pVTZ and in $\left.\mathrm{ref}^{27}\right)$. Experiments probing in the singlet adiabatic dissociation threshold range using a $193 \mathrm{~nm}(6.424 \mathrm{eV})$ pulse began more than three decades ago ${ }^{28}$; The nascent $\mathrm{C}_{2} \mathrm{H}$ was measured to have energies peaking around $0.25 \mathrm{eV}$ (and up to $0.69 \mathrm{eV}$ ), but with little near $0 \mathrm{eV}$, suggesting that little internal conversion occurs ${ }^{29}$; the trans is active at the time of excitation and likely to have this configuration at the time of dissociation ${ }^{5}$.

Fuji $e a^{20}$ posited a non-radiative relaxation channel opening above $6.2 \mathrm{eV}$, which coincides with the calculated $\mathrm{S}_{2}$ barrier towards dissociation or $\mathrm{S}_{0}$ crossing ${ }^{13}$. The vibrational UV-IR double resonance spectra progressions in the 6.2-7.1 were assigned to trans and antisymmetric stretch vibrations. Zhang et al posited a dissociation threshold above $193.3 \mathrm{~nm}$ $(6.41 \mathrm{eV})$ leading to an efficient adiabatic ${ }^{2} \Pi$ production when exceeded, in agreement with Cui and Morokuma ${ }^{18}$. Schmid et al ${ }^{1,30}$ excited up to $7.02 \mathrm{eV}$ and observed two dissociation channels they assign to $X^{2} \Sigma^{+}$and $\tilde{A}^{2} \Pi$ states, with $\mathrm{CH}$ rupture being increased by rovibrational pre-excitation. Ganot et $a l^{21,31}$ probed around $6.3 \mathrm{eV}$, just below the adiabatic dissociation threshold 6.4, to study the dissociation via the nonadiabatic $\tilde{A} / \tilde{B}$ pathway. They found transitions involving trans modes and dissociation pathway leading to the $X^{2} \Sigma^{+}$state enhanced.

\section{Model Hamiltonian}

\subsection{Vertical excitation Energies}

Table 2 shows vertical excitation energies obtained using two methods, complete active space (CAS) with second order perturbation theory corrections (PT2) and equation-of-motion coupled cluster singles and doubles (EOM-CCSD), with increasing basis set size. The active space for the former was obtained by performing single point calculations with different low-energy orbitals, including up to 17 orbitals for any given calculation. By weighting the contribution that every orbital has on $\mathrm{N}$ states proportionally to the energy of the states which they describe, it is possible to reduce the active space to those orbitals that best describe the lowest $\mathrm{N}$ states. The orbitals thus obtained were in agreement with those of EOMCCSD calculations at the same basis set level.

For an MP2 optimised geometry (cc-pVDZ), the following symmetry and energy ordering in a aug-cc-pVTZ, HF calculation labels the active space orbitals: $4 \mathrm{~A}_{g}, 1 \mathrm{~B}_{3 u}, 2 \mathrm{~B}_{3 u}$, $1 \mathrm{~B}_{2 u}, 2 \mathrm{~B}_{2 u}, 3 \mathrm{~B}_{1 u}, 4 \mathrm{~B}_{1 u}, 1 \mathrm{~B}_{2 g}, 2 \mathrm{~B}_{2 g}$. Geometry optimisations were done with Gaussian $03^{32}$ and excited state calculations with Molpro $09^{33}$ For a given basis, neither a second or third order-perturbation changes state ordering and the results agree with those of EOM-CCSD calculations. The inclusion of augmented diffuse functions, however, has the effect of swapping the order of degenerate states $\Pi_{u}$ with $\Pi_{g}$. Finally, inclusion 

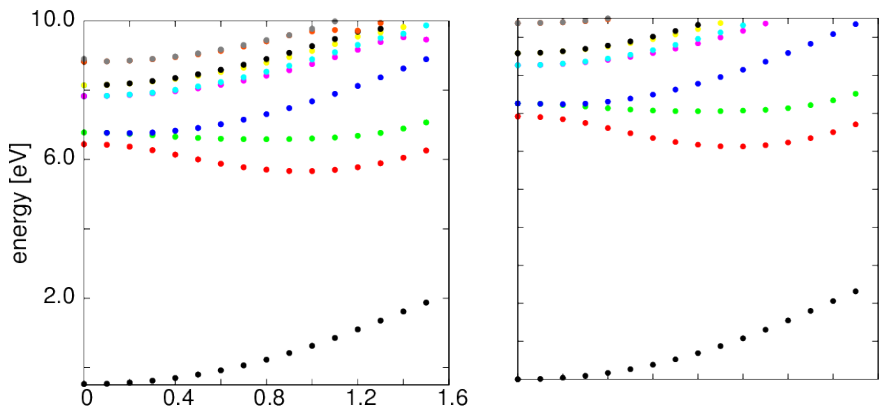

Fig. 1 Comparison of cuts along $\mathrm{CCH}$ angle using left: EOM-CCSD/aug-cc-pV(q+d)Z, right: EOM-CCSD/aug-cc-pVTZ

of polarisable $\mathrm{pV}(\mathrm{q}+\mathrm{d}) \mathrm{Z}$ functions further lowers the energy. Performing a cut on the PES, Fig. 1 shows that the main effect of polarisable functions is to lower the absolute energies of the states without perturbing their topology and relative energies. Since the difference between the relative energies of excited states is slight, for practical purposes, models were fitted to an EOM-CCSD/aug-cc-pVTZ energies. Some of the optimised geometries or critical points on excited state surfaces in the literature are given in Tab 1 and were also mentioned in the introduction.

\subsection{Choice of coordinates}

Properly modelling the potential landscape of acetylene requires a natural description of the cis-trans isomerisation and out-of-plane torsion as a function of the dissociating coordinates. It is clear that normal modes are inadequate for the description of such excited state critical points; their rectilinear character makes them restrictive on the kind of molecular distortions one can easily describe (typically small and bound displacements) without giving rise to rapidly changing state energies that make a low order polynomial model prohibitive. Jacobi $^{34}$ and valence ${ }^{35}$ curvilinear coordinates are offered in the literature but have disadvantages for the construction of vibronic models built out of products of $1 \mathrm{D}$ operators. They require the molecule to be non-linear for the dihedral angle to be defined, resulting in strong correlation of the energy along this degree of freedom (DOF) with regard to the others. Additionally, this same feature reduces the symmetry of the coordinates from $\mathrm{D}_{\infty \mathrm{h}}$ to $C_{2 h}$ which allows a much larger number of parameters into the model, complicating the fitting procedure. Unable to use $\Sigma_{g}^{+}$symmetry adapted basis, they becomes prohibitive if we hope to describe all internal DOF and multiple states.

The above implications suggest we would like curvilinear coordinates that ideally span as many $\mathrm{D}_{\infty \mathrm{h}}$ irreps as possible so as to reduce the terms that enter into our model. A solution is to use the same kinds of displacements that the

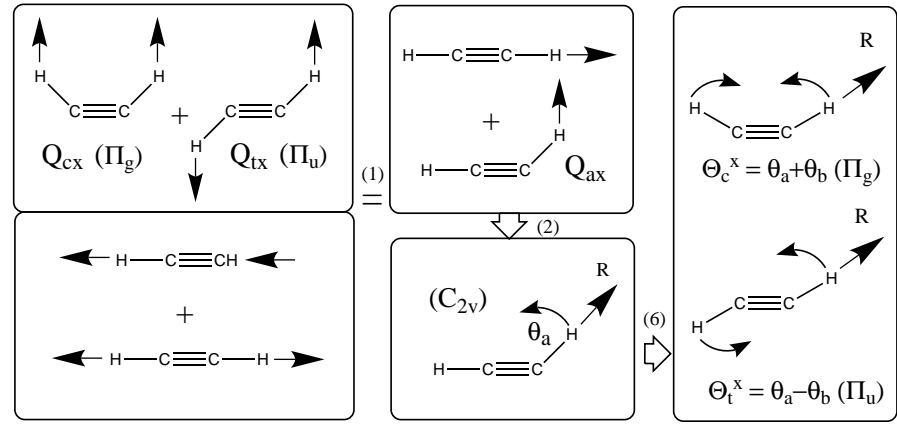

Fig. 2 The steps taken to obtain curvilinear coordinates starting from normal coordinates. Only coordinates in a single plane are shown. Numbers in brackets refer to the equations in text.

$\mathrm{D}_{\infty \mathrm{h}}$ normal coordinates have, but involving $\mathrm{CCH}$ angles and radial (valence like) coordinates. To obtain such coordinates, the Cartesian coordinates were mass-scaled relative to the reference geometry: $\left(x_{i}-x_{i}^{0}\right) \rightarrow \frac{1}{\sqrt{m_{i}}} \tilde{x}_{i}$, and by diagonalizing the Hessian a set of normal coordinates were obtained (see Tab 3), four of which belong to degenerate $\Pi_{\mathrm{g}}$ and $\Pi_{\mathrm{u}}$ IrReps and which describe trans and cis displacements.

The next steps taken are diagrammatically summarised in Fig. 2. Rotations of these four normal coordinates were performed to obtain a set of quasi-Cartesian coordinates describing the position of the hydrogen atoms:

$$
\left(\begin{array}{l}
Q_{a y} \\
Q_{b y}
\end{array}\right)=\frac{1}{\sqrt{2}}\left(\begin{array}{cc}
1 & -1 \\
1 & 1
\end{array}\right)\left(\begin{array}{l}
Q_{c y} \\
Q_{t y}
\end{array}\right)
$$

Where $Q_{a y}$ refers to the quasi y-coordinate of hydrogen atom 'a' (similar definition for $Q_{b y}$ ) and $Q_{c y}$ and $Q_{t y}$ refer to the cis and trans normal coordinates along the yz-plane. A similar operation is done for the xz-plane coordinates, $Q_{c x}$ and $Q_{t x}$, and for the symmetric and antisymmetric stretches, $Q_{s s}$ and $Q_{a s}$, to give single atom stretches along the z-axis. The only difference between the Cartesian coordinates and these is that the other bond distances change slightly so as to fix the centre of mass and to maintain the linear space of linear rotations and translations outside our coordinate space. Since we are using mass-scaled normal coordinates, the KE operator is invariant with respect to rotations.

From here a spherical polar transformation of each $\mathrm{H}$ atom quasi-Cartesian coordinates was performed to obtain two sets of quasi-spherical polar coordinates:

$$
\begin{aligned}
& Q_{a x}=R_{a} \cdot \cos \left(\theta_{a}\right) \\
& Q_{a y}=R_{a} \cdot \sin \left(\theta_{a}\right) \cdot \sin \left(\phi_{a}\right) \\
& Q_{a z}=R_{a} \cdot \sin \left(\theta_{a}\right) \cdot \cos \left(\phi_{a}\right)-Q_{0}^{z}
\end{aligned}
$$

These are defined in such a way that the linear minima in the ground state lies at $\theta_{a}=\frac{\pi}{2}, \phi_{a}=\pi, R_{a}=Q_{0}^{z}$. A similar set of 


\begin{tabular}{cccccc}
\hline State & $\mathrm{A}$ & $\mathrm{B}$ & $\mathrm{C}$ & $\mathrm{D}$ & $\mathrm{E}$ \\
\hline $\mathrm{S}_{0}$ & $-77.164\left(\Sigma_{g}^{+}\right)$ & $-77.091\left(\Sigma_{g}^{+}\right)$ & $-77.175\left(\Sigma_{g}^{+}\right)$ & $-77.193\left(\Sigma_{g}^{+}\right)$ & $-77.171\left(\Sigma_{g}^{+}\right)$ \\
$\mathrm{S}_{1}$ & $7.025\left(\Sigma_{u}^{+}\right)$ & $7.596\left(\Sigma_{u}^{+}\right)$ & $7.213\left(\Sigma_{u}^{+}\right)$ & $7.213\left(\Sigma_{u}^{+}\right)$ & $7.367\left(\Sigma_{u}^{+}\right)$ \\
$\mathrm{S}_{2}$ & $7.342\left(\Delta_{u}\right)$ & $7.990\left(\Delta_{u}\right)$ & $7.538\left(\Delta_{u}\right)$ & $7.534\left(\Delta_{u}\right)$ & $7.730\left(\Delta_{u}\right)$ \\
$\mathrm{S}_{3}$ & $7.343\left(\Delta_{u}\right)$ & $8.035\left(\Delta_{u}\right)$ & $7.538\left(\Delta_{u}\right)$ & $7.534\left(\Delta_{u}\right)$ & $7.730\left(\Delta_{u}\right)$ \\
$\mathrm{S}_{4}$ & $8.117\left(\Pi_{u}\right)$ & $9.788\left(\Pi_{g}\right)$ & $8.375\left(\Pi_{u}\right)$ & $8.415\left(\Pi_{u}\right)$ & $9.536\left(\Pi_{g}\right)$ \\
$\mathrm{S}_{5}$ & $8.117\left(\Pi_{u}\right)$ & $9.788\left(\Pi_{g}\right)$ & $8.375\left(\Pi_{u}\right)$ & $8.415\left(\Pi_{u}\right)$ & $9.536\left(\Pi_{g}\right)$ \\
$\mathrm{S}_{6}$ & $8.520\left(\Pi_{g}\right)$ & $10.638\left(\Pi_{u}\right)$ & $8.736\left(\Pi_{g}\right)$ & $8.776\left(\Pi_{g}\right)$ & $10.118\left(\Pi_{u}\right)$ \\
$\mathrm{S}_{7}$ & $8.520\left(\Pi_{g}\right)$ & $10.638\left(\Pi_{u}\right)$ & $8.736\left(\Pi_{g}\right)$ & $8.776\left(\Pi_{g}\right)$ & $10.118\left(\Pi_{u}\right)$ \\
$\mathrm{S}_{8}$ & $\mathrm{n} / \mathrm{a}$ & $11.768\left(\Sigma_{g}^{-}\right)$ & $9.476\left(\Delta_{\mathrm{g}}\right)$ & $9.404\left(\Delta_{\mathrm{g}}\right)$ & $11.578\left(\Sigma_{g}^{-}\right)$ \\
$\mathrm{S}_{9}$ & $\mathrm{n} / \mathrm{a}$ & $13.106\left(\Delta_{\mathrm{g}}\right)$ & $9.476\left(\Delta_{\mathrm{g}}\right)$ & $9.404\left(\Delta_{\mathrm{g}}\right)$ & $12.249\left(\Delta_{\mathrm{g}}\right)$ \\
\hline
\end{tabular}

Table 2 Vertical excitation energy (eV) comparison between CAS(6,9)+PT2 and EOM-CCSD with different basis-sets. $\mathrm{S}_{0}$ in Hartree A) CAS(6,9)+PT2//aug-cc-pVTZ B) CAS(6,9)+PT2//pVTZ C) EOM-CCSD//aug-cc-pVTZ D) EOM-CCSD//aug-cc-pV(q+d)Z E) $\mathrm{EOM}-\mathrm{CCSD} / \mathrm{pVTZ}$

\begin{tabular}{cccc}
\hline Mode & Motion & Symmetry & $\omega\left(\mathrm{cm}^{-1}\right)$ \\
\hline$\tilde{v}_{1,2}\left(Q_{t x, t y}\right)$ & - & $\Pi_{g}$ & 557.8506 \\
$\tilde{v}_{3,4}\left(Q_{c x, c y}\right)$ & & $\Pi_{u}$ & 749.5317 \\
$\tilde{v}_{5}\left(Q_{c c s}\right)$ & & $\Sigma_{g}^{+}$ & 1965.0605 \\
$\tilde{v}_{6}\left(Q_{a s}\right)$ & & $\Sigma_{u}^{+}$ & 3455.1418 \\
$\tilde{v}_{7}\left(Q_{s s}\right)$ & $-0-0$ & $\Sigma_{g}^{+}$ & 3540.0541 \\
\hline
\end{tabular}

Table 3 Harmonic Frequencies. Obtained from an MP2 calculation with an cc-pVTZ basis.

\begin{tabular}{lllllll}
\hline State & Char & $\mathrm{D}_{\infty \mathrm{h}}$ & $C_{2 h}$ & $C_{2 \mathrm{v}}$ & $C_{s}$ & $C_{\infty \nu}$ \\
\hline $\mathrm{S}_{0}$ & & $\Sigma_{g}^{+}$ & $A_{g}$ & $A_{1}$ & $A^{\prime}$ & ${ }^{2} \Sigma^{+}$ \\
$\mathrm{S}_{1}$ & $1 \pi_{g}$ & $\Sigma_{u}^{-}$ & $A_{u}$ & $A_{2}$ & $A^{\prime \prime}$ & ${ }^{2} \Pi$ \\
$\mathrm{S}_{2} / \mathrm{S}_{3}$ & $1 \pi_{g}$ & $\Delta_{u}$ & $A_{u} \otimes A_{u}$ & $A_{2} \otimes B_{1}$ & $A^{\prime \prime} \otimes A^{\prime}$ & ${ }^{2} \Pi$ \\
$\mathrm{S}_{4} / \mathrm{S}_{5}$ & $3 \mathrm{~s} \sigma_{g}$ & $\Pi_{u}$ & $B_{u} \otimes B_{u}$ & $B_{2} \otimes B_{1}$ & $A^{\prime \prime} \otimes A^{\prime}$ & \\
$\mathrm{S}_{6} / \mathrm{S}_{7}$ & $3 \mathrm{p} \sigma_{u}$ & $\Pi_{g}$ & $B_{g} \otimes B_{g}$ & $B_{1} \otimes B_{2}$ & $A^{\prime} \otimes A^{\prime \prime}$ & \\
$\mathrm{S}_{8} / \mathrm{S}_{9}$ & $3 \mathrm{~s} \pi_{u}$ & $\Delta_{\mathrm{g}}$ & $A_{g} \otimes A_{g}$ & $A_{1} \otimes A_{2}$ & $A^{\prime} \otimes A^{\prime \prime}$ & \\
\hline
\end{tabular}

Table 4 Singlet state and symmetry correlation tables. Character of Rydberg states is based on Ref $^{11}$. Last column refers to the doublet state which correlates adiabatically via dissociation. coordinates are obtained for atom ' $b$ '. For these coordinates, the resulting $\mathrm{KE}$ operator is potentially the simplest form this system could have: it consists of a pair of angular momentum operators, radial KE terms and a normal KE term for $Q_{c c s}$ :

$$
2 \cdot \hat{T}=\hat{p}_{i}^{2}+\frac{\hat{L}_{i}^{2}}{R_{i}^{2}}+\nabla_{c c s}^{2}, \quad i=a, b
$$

where

$$
\begin{aligned}
\hat{L}_{i}^{2} & =\frac{1}{\sin \left(\theta_{i}\right)} \frac{\partial}{\partial \theta_{i}} \sin \left(\theta_{i}\right) \frac{\partial}{\partial \theta_{i}}+\frac{1}{\sin ^{2} \theta_{i}} \frac{\partial^{2}}{\partial \phi_{i}^{2}} \\
\hat{p}_{i}^{2} & =\frac{1}{R_{i}^{2}} \frac{\partial}{\partial R_{i}} R_{i}^{2} \frac{\partial}{\partial R_{i}}
\end{aligned}
$$

This operator can be simplified further by factoring the radial part of the wavefunction with $R_{i}, \Phi_{R}\left(R_{i}\right) \equiv R_{i} \cdot \Phi\left(R_{i}\right)$. In this way the radial kinetic energy term takes an "equivalent onedimensional" form ${ }^{36}$ :

$$
2 \cdot \hat{T}=\left(\frac{1}{i} \frac{\partial}{\partial R_{j}}\right)^{2}+\frac{\hat{L}_{j}^{2}}{R_{j}^{2}}+\nabla_{c c s}, \quad j=a, b
$$

These coordinates posses $\mathrm{C}_{2 \mathrm{v}}$ symmetry. By operating with the analogous but inverse rotation matrix of equation 1 on the angular coordinates, a coordinate representation is recovered that spans the $\mathrm{D}_{\infty \mathrm{h}}$ point group:

$$
\left(\begin{array}{l}
\Theta_{c}^{x} \\
\Theta_{t}^{x}
\end{array}\right)=\frac{1}{\sqrt{2}}\left(\begin{array}{cc}
1 & 1 \\
-1 & 1
\end{array}\right)\left(\begin{array}{l}
\theta_{b} \\
\theta_{a}
\end{array}\right)
$$

where $\Theta_{t}^{x}$ and $\Theta_{c}^{x}$ correspond to trans and cis angular motion along the zx-plane. With the same treatment for the zyplane coordinates, $\left\{\Theta_{t}^{x}, \Theta_{t}^{y}\right\}$ and $\left\{\Theta_{c}^{x}, \Theta_{c}^{y}\right\}$ form a basis for $\Pi_{u}$ and $\Pi_{g}$ IrReps respectively. Crucially, these coordinates give a much more accurate description of excited state critical points than do normal coordinates. Although $\left\{\Theta_{t}^{x}, \Theta_{t}^{y}\right\}$ and 
$\left\{\Theta_{c}^{x}, \Theta_{c}^{y}\right\}$ allow us to use the $\Sigma_{g}^{+}$symmetry adapted matrices ${ }^{9}$, and were indeed used to construct the model within the $\Theta_{t}^{x}$ and $\Theta_{c}^{x}$ space, subsequently returning to $\{\theta, \phi\}$ coordinates also proved advantageous since these leave the KE operator in the simple form given in Eq. 5. One could have conceivably used the valence coordinates to obtain an analogous set of coordinates, but the resulting transformed kinetic energy operator would have been more complicated.

\subsection{Potential surface and Fitting}

Due to symmetry considerations, combined displacements along several of these high symmetry coordinates can result in identical potential energy along some other displacement. For example, since $\phi_{a}+\theta_{b}$ and $\phi_{b}+\theta_{a}$ are related by a reflection, they are physically (energetically) indistinguishable. Single energy points were calculated in physically distinct regions of the potential energy landscape of the trans and cis coordinate subspace, $\Theta_{c \mid t}^{x \mid y}$, forming a basis for $\Pi_{g / u}$ IrReps. $\Sigma_{g}^{+}$symmetry adapted polynomials that form a matrix representation of the diabatic potential were fitted using a genetic algorithm tailored for these optimisation tasks ${ }^{37}$.

The resulting fitted potential energy model for the two RT $\left\{\Theta_{c \mid t}^{x \mid y}\right\}$ pairs of degenerate $\Pi_{g / u}$ coordinates, with its calculated absorption spectra, was presented elsewhere ${ }^{9}$. The first three states show stabilisation away from the linear geometry which must arise from pJT coupling to higher lying states ${ }^{16}$. Ten states were required to fit satisfactorily the RT coordinates, having used 78 symmetry adapted basis function. After rotating the model back to the $\mathrm{C}_{2 \mathrm{v}}$ coordinates (to keep the simple KE form), these four dimensions alone contribute 337 monomials into the potential energy matrix. Were lower or no symmetry to be used, the number of allowed terms entering the fitting procedure for such a model would become prohibitive. Lest one argues the model to be over-parametrised, an 8 state model was attempted with no satisfactory fitting. The model allows for a proper free dihedral rotation to occur.

To describe dissociation it was necessary to fit functions that adequately describe the potential along $R_{a \mid b}$ coordinates and which correlate these to the others. The model and ab initio adiabatic energies along these are shown in Fig. 3.

Since the ground state is significantly separated from the rest of the states, and it was adequately represented by 1D Morse potential functions, it is excluded in Fig. 3 in all but these two panels. $R_{a \mid b}$ were not rotated to the higher $\Sigma^{+/-}$symmetries; being $\mathrm{CH}$ radial coordinates, these are the most relevant coordinates for dissociation and were fitted with Morse functions. Along these dissociating coordinates, states $4 \Pi_{g}$ are shaped by strong diabatic coupling to some other high lying repulsive states (of $\Pi_{g}$ or $\Delta_{\mathrm{g}}$ symmetry) resulting in an adiabatic barrier-less dissociating surface (see top left, Fig. 3).
Since we can only expect the density of states to increase at higher energies and compound the complexity of interactions, the diabatic coupling responsible for this dissociative $\Pi_{g}$ state was approximated by a function which 'folds' the coupling with the higher lying state into the diagonal element:

$$
W_{11}=\frac{1}{2}\left\{v_{M}+v_{d}-\sqrt{\left(v_{M}-v_{d}\right)^{2}+4[\alpha \tanh (\beta Q)]^{2}}\right\}
$$

where $v_{M}$ is a Morse potential function, $v_{d}$ a decaying exponential and $\alpha \tanh (\beta Q)$ describes the coupling to higher lying states; as the coordinate dissociates the coupling reaches some asymptotic value. The cost of using this approximate function is that it fails to properly describe the correct topology when moving across several DOF (presumably along which the missing higher lying states would have appropriately correlated the surfaces). Specifically, when correlating both dissociative, $R_{a}$ and $R_{b}$, coordinates the model surface does not rise as quickly as it should and when correlating $Q_{c c s}$ with $R_{a}$ the dissociating channel does not fall as quickly as it should ( bottom right panels in Fig. 3).

Additional ad-hoc functions were also used to fits these spaces. For $\mathrm{j}=1,2$, indexing degenerate state $\Pi_{g}$, between $R_{a}$ and $R_{b}$;

$$
\begin{aligned}
& W_{j \Pi_{g}, j \Pi_{g}}=\gamma_{R_{a}, R_{b}} \cdot \Theta^{+}\left(R_{a}-0\right) \Theta^{+}\left(R_{b}-0\right) \cdot R_{a} R_{b} \\
& W_{j \Pi_{g}, j \Pi_{g}}=\varepsilon_{R_{a}, R_{b}} \cdot\left\{\Theta^{-}\left(R_{a}-0\right) \Theta^{+}\left(R_{b}-0\right) \cdot R_{a} R_{b}+\right. \\
& \left.\Theta^{+}\left(R_{a}-0\right) \Theta^{-}\left(R_{b}-0\right) \cdot R_{a} R_{b}\right\}
\end{aligned}
$$

and between $Q_{c c s}$ and $R_{a}$;

$$
\begin{aligned}
W_{j \Pi_{g}, j \Pi_{g}}= & \beta_{R_{a}, c c s} \cdot \Theta^{-}\left(R_{b}-0\right) \cdot \tanh \left(\lambda_{R_{b}} R_{b}\right) \cdot \\
& \tanh \left(\lambda_{R_{a}} R_{a}\right) \cdot \frac{1}{2}\left(\tanh \left(\lambda_{c c s}\left(Q_{c c s}-Q_{c c s}^{0}\right)\right)\right.
\end{aligned}
$$

Bilinear parameters $\left(\gamma_{R_{a}, R_{b}}\right.$ and $\left.\varepsilon_{R_{a}, R_{b}}\right)$ correlate $R_{a}$ and $R_{b}$ with different values along different quadrants. Parameters $\beta_{R_{a}, c c s}, \lambda_{R_{b}}, \lambda_{c c s}, Q_{c c s}^{0}$ determine the functions correlating $Q_{c c s}$ with $R_{a} . \quad \Theta^{+/-}$are forward/backward step functions. Although the first functions are non-differentiable at $R_{a \mid b}=0$ (albeit continuous), they do not break symmetry and since the multi configurational time dependent hartree algorithm (MCTDH), used for the quantum propagation of this model, uses a grid representation, it does not pose a problem. The top right panels in Fig. 3 show the cis and trans displacement correlating mode $Q_{c c s}\left(Q_{c c s}\right.$ frozen at the $\mathrm{S}_{1}$ minima along that DOF); these cuts approximately show the two important $S_{1}$ minima.

Finally, functions that correlate the four angular RT coordinates to the radial $R_{a \mid b}$ coordinates were fitted to single point energies along the same selection of physically distinct displacements described earlier (and shown in ref $^{9}$ ) but including $R_{a \mid b}$ contributions. Polynomials are inappropriate for the 


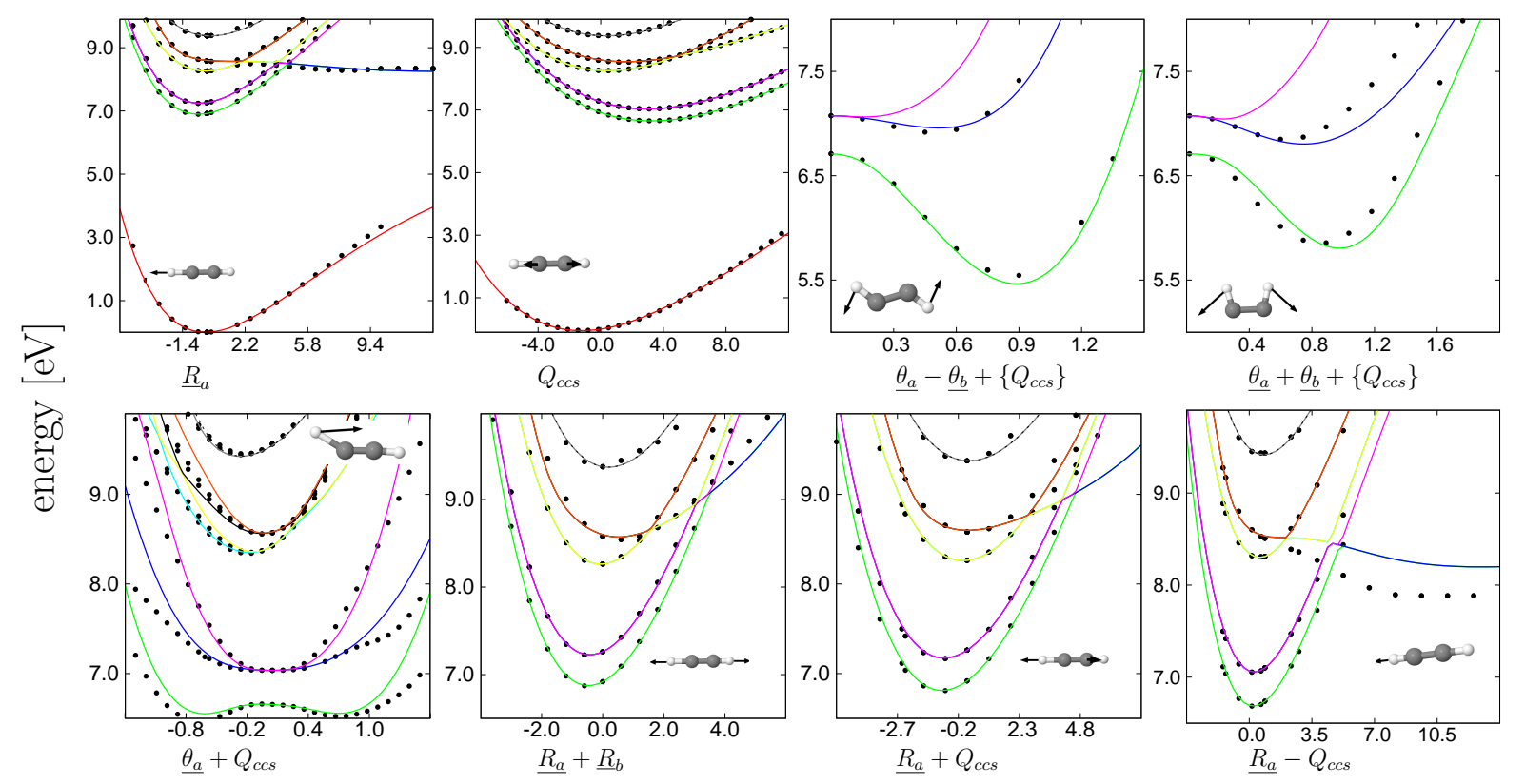

Fig. 3 Potential energy surface cuts of the fitted model to calculated points of the excited states correlating $Q_{c c s}$ to $R$ or $\theta$ coordinates for $\mathrm{S}_{1}-\mathrm{S}_{9}$. Since no terms correlate coordinates in $\mathrm{S}_{0}$, only the first two top left frames show $\mathrm{S}_{0}$. Curly brackets mean the coordinate is kept constant but not at the reference geometry. Energy in eV, coordinates in $\AA$ amu $]^{\frac{1}{2}}$. The underline indicates which coordinate is shown in plot (for mixed angular/radial/normal vectors). From left to right: first row: $\left(R_{a}\right),\left(Q_{c c s}\right)$, trans $\left(\underline{\theta_{a}}-\theta_{b}+\left\{Q_{c c s}\right\}\right)$, cis $\left(\underline{\theta_{a}}+\theta_{b}+\left\{Q_{c c s}\right\}\right) ;$ second row: $\left(\underline{\theta_{a}}+Q_{c c s}\right),\left(\underline{R_{a}}+\underline{R_{b}}\right),\left(\underline{R_{a}}+Q_{c c s}\right),\left(\underline{R_{a}}-Q_{c c s}\right)$

description of the asymptotic behaviour of dissociating coordinates. The following functions adequately fit the surfaces at the $R_{a \mid b} \rightarrow \infty$ regions.

For pJT coupling elements:

$$
W_{i j}\left(R_{a}, \theta_{a}, \phi_{a}\right)=\gamma \cdot \tanh \left(\lambda \cdot R_{a}\right) \cdot \sin \left(\alpha \cdot \theta_{a}\right) \cdot \mathrm{G}\left(\beta, \phi_{a}\right)
$$

where

$$
\begin{aligned}
\mathrm{G}\left(\beta, \phi_{a}\right)= & \frac{1}{4} \cdot\left(\cos \left(\beta \cdot \phi_{a}\right)+3\right) \cdot \Theta^{+}\left(\phi_{a}-\frac{\pi}{\beta}\right) \cdot \Theta^{-}\left(\phi_{a}+\frac{\pi}{\beta}\right)+ \\
& \frac{1}{2} \cdot\left(\Theta^{-}\left(\phi_{a}-\frac{\pi}{\beta}\right)+\Theta^{+}\left(\phi_{a}+\frac{\pi}{\beta}\right)\right)
\end{aligned}
$$

with $\gamma, \lambda, \alpha$ and $\beta$ parameters to be optimised. Linear terms corresponding to radial displacements have been replaced by tanh functions, and even/odd polynomial functions were replaced by even/odd trigonometric functions. It is worth mentioning that these function obey $\mathrm{D}_{2 h}$ symmetry operations and one can make it obey $\mathrm{D}_{\infty \mathrm{h}}$ constraints. For example, to enforce constraints on $\Pi \otimes \Delta$ coupling functions:

$$
\begin{aligned}
+W_{\Pi_{x}, \Delta_{y}}\left(R_{a}, \theta_{a}, \phi_{a}\right) & =-W_{\Pi_{x}, \Delta_{y}}\left(R_{b}, \theta_{b}, \phi_{b}\right) \\
=+W_{\Pi_{x}, \Delta_{x}}\left(R_{a}, \phi_{a}, \theta_{a}\right) & =-W_{\Pi_{x}, \Delta_{x}}\left(R_{b}, \phi_{b}, \theta_{b}\right) \\
=-W_{\Pi_{y}, \Delta_{x}}\left(R_{b}, \theta_{b}, \phi_{b}\right) & =+W_{\Pi_{y}, \Delta_{x}}\left(R_{a}, \theta_{a}, \phi_{a}\right) \\
= & +W_{\Pi_{y}, \Delta_{y}}\left(R_{b}, \phi_{b}, \theta_{b}\right)=-W_{\Pi_{y}, \Delta_{y}}\left(R_{a}, \phi_{a}, \theta_{a}\right)
\end{aligned}
$$

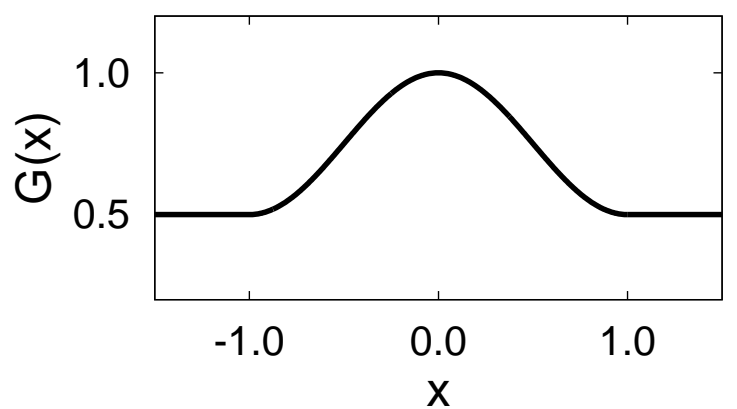

Fig. 4 The $\mathrm{G}(\beta, x)$ function of Eq. (9), with $\beta=1$

$W_{i j}$ as defined above (and based on the procedure described in $\left.\operatorname{ref}^{9}\right)$.

For diagonal inter-state diabatic coupling:

$W_{i i}\left(R_{a}, \theta_{a}, \phi_{a}\right)=\gamma \cdot \tanh \left(\lambda \cdot R_{a}\right) \cdot 2\left(1-\cos \left(\alpha \cdot \theta_{a}\right)\right) \cdot \mathrm{G}\left(\beta, \phi_{a}\right)$

with similar symmetry conditions and parameters as the aforementioned coupling elements. $G$ (shown in Fig. 4) ensures that the functions do not couple too strongly when, say, simultaneous displacements along $\theta_{a}$ and $\phi_{a}$ occur: $W_{i i}\left(R_{a}, \theta_{a}, \phi_{a}\right)+W_{i i}\left(R_{a}, \phi_{a}, \theta_{a}\right)$ approximately results in the same value as moving on either $\theta_{a}$ or $\phi_{a}$ alone). The model in MCTDH input form is provided as supplementary information.

The resulting fitted functions along some interesting cuts 

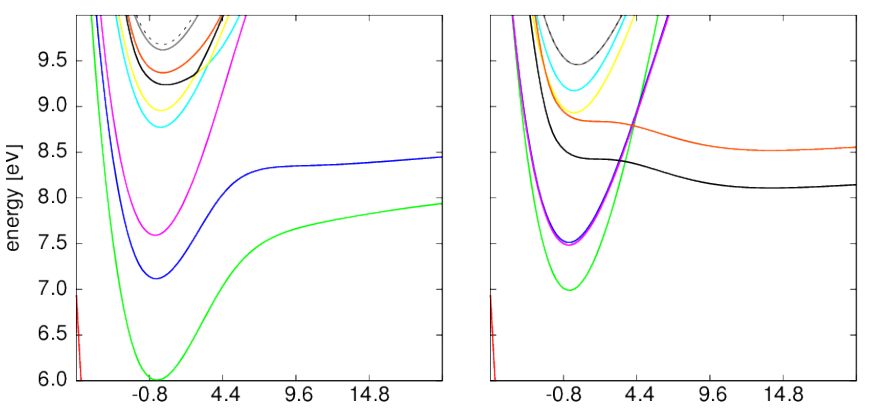

Fig. 5 Qualitative comparison of the adiabatic (left) and diabatic (right) intra-state element surfaces along a dissociating coordinate starting from a trans geometry.

describing out-of-plane, cis and trans geometries leading to dissociation, are shown in Figs. 5 and 6. Since $Q_{c c s}$ is kept at the ground state reference geometry, the cis and trans minima shown for $\mathrm{S}_{1}$ in the figure are not global and lie approximately $1 \mathrm{eV}$ above the actual value. They are nevertheless qualitatively similar, as can be seen by comparing the cuts involving angles in Fig. 3 to those in Fig. 6; $Q_{c c s}$ only weakly couples to the other coordinates in $\mathrm{S}_{1}-\mathrm{S}_{3}$. Given the coordinate system used, the geometries of the $\mathrm{C}_{2} \mathrm{H}$ moiety after dissociation in the different cuts in Fig. 6 are slightly different. The fitting procedure was not trivial and a genetic algorithm was used ${ }^{37}$, involving $\sim 40$ optimisation parameters. Fig. 5 compares a cut along some trans dissociation channel in the diabatic and adiabatic representations; the diabatic dissociative $6 \Pi_{g}$ states get mixed strongly (via pJT coupling) with $1 \Sigma_{u}^{-}$and $2 \Delta_{u}$ states so that they instead become the dissociative channel.

A significant approximation in this model is that no coupling to the ground state was included, so that non-radiative relaxation to the ground state will not be reproduced (as observed experimentally ${ }^{1,31}$ ). Theoretically, such a possible pathway was found by Cui and Morokuma ${ }^{18}$ along a geometry with strong dihedral and angle values $\left(\sim 90^{\circ}\right.$ for both angles) with a $\mathrm{CH}$ bond length of $2.111 \AA$, very close to dissociation. Such a displacement is poorly described by the present curved modes. Coupling along such a crossing required the involvement of many degrees of freedom and was not adequately fitted with these coordinates.

Another potential crossing was suggested by Laruelle et $a l^{11}$ in a figure showing an avoided crossing along the $\mathrm{CH}$ dissociation coordinate. Based on the electronic structure calculations shown in Tab 2 we suggest their symmetry label assignment to $\Pi_{g}$ and $\Pi_{u}$ states were incorrectly swapped; at the $\mathrm{D}_{\infty \mathrm{h}}$ geometry, it is $\Pi_{g}$ which is the dissociative coordinate. To certify whether such a crossing occurs or not, a high accuracy calculation was performed using a aug-cc-pVTZ basis with 3 extra even-tempered functions (ratio $=2.5)^{33}$ on the dissociating carbon and hydrogen atoms were added. Using $C_{2 \mathrm{v}}$ symmetry and starting at a $1.713 \AA$ bond length, a CAS $(8,8)$ energy was obtained after several calculations with different active orbitals to test for their contribution to the first three excited states. From this active space, RS2C and MR CI (cluster corrected Pople/Davidson) calculations were performed, plotting the hydrogen dissociation coordinate (Fig. 7). No crossing is found between $S_{0}-S_{1}$ at the linear geometry with these methods. No crossing was found along any other geometry with the level of theory used for the model construction. Therefore, in this model no states beyond the $\mathrm{S}_{1} / \mathrm{S}_{2}$ asymptotic dissociation regions ( $>1.9 \mathrm{CH} \AA$ ) cross with these.

To explore the adiabatic potential energy landscape of this model, simplex optimisations were performed with initial geometries set at different points in an equidistant grid across all 7 coordinates. Fig. 8 shows the histograms of the minima obtained. They give some qualitative insight into the basin landscape of the model. Some sampled energies and geometries are given in Tab 1; these are representative of the frequency with which these were found based on a few samples chosen and should not be taken as precise (a more holistic picture will be provided in section 3). The top right panel shows the trans and cis minima basins starting at around $\sim 5.4$ and $\sim 5.6 \mathrm{eV}$ respectively. The third peak at $\sim 5.8$ contains a mixture of cis and bent geometries, with out-of-plane geometries for the higher energies. The $S_{2}$ surface (bottom right panel) has three peaks - the $\sim 6.58$ peak corresponds to strong cis (angles close to 90 ) with out-of-plane (cisoid), $\sim 6.65$ peak has weaker $c i s$ and out-of-plane mixtures and the $\sim 6.78$ peak is similar to the former but with bent (only one $\mathrm{CH}$ bond) geometries also present. These geometries agree qualitatively with the findings of Schubert et $\mathrm{al}^{27}$ and some representative geometries given in Tab 1. For $S_{3}$ only basins of out-of-plane cisoid geometries with closely similar energies where found. A wavefunction relaxation in the $S_{1}$ state gives energies of $6.024 \mathrm{eV}$ $=5.4 \mathrm{eV}+\mathrm{ZPE}$, using ZPE in line with the literature ${ }^{18}$.

For the left panels of the figure, one of the dissociative coordinates was stretched to $1.9 \AA$ and frozen during the optimisation to give some perspective on the landscape near dissociation. For these stretched coordinates, the $\mathrm{S}_{1}$ minima (top left) is posited to be at $\sim 6.5 \mathrm{eV}$ and have a mixture of trans and cis conformations (provided in Tab 1 as $d$-trans/cis). There are a significantly more trans conformations for the lowest energy region, with more cis as one approaches $7.5 \mathrm{eV}$. This is likely due to the fact the $\mathrm{H}$ atom continues to have an effect on the potential energy (at $\infty$ one would expect equal amounts of conformers). The second peak starting $\sim 7.8 \mathrm{eV}$ is mostly populated by cis conformations. Calculations on critical point optimisation ${ }^{18}$ suggest cis geometries to be involved during the transition towards dissociation. Finding more trans conformations at the lowest dissociation energy region of this model would suggest trans to be the lowest energy path and this is found to be the case in dynamics simulations, somewhat in disagreement with the aforementioned critical point study. For 


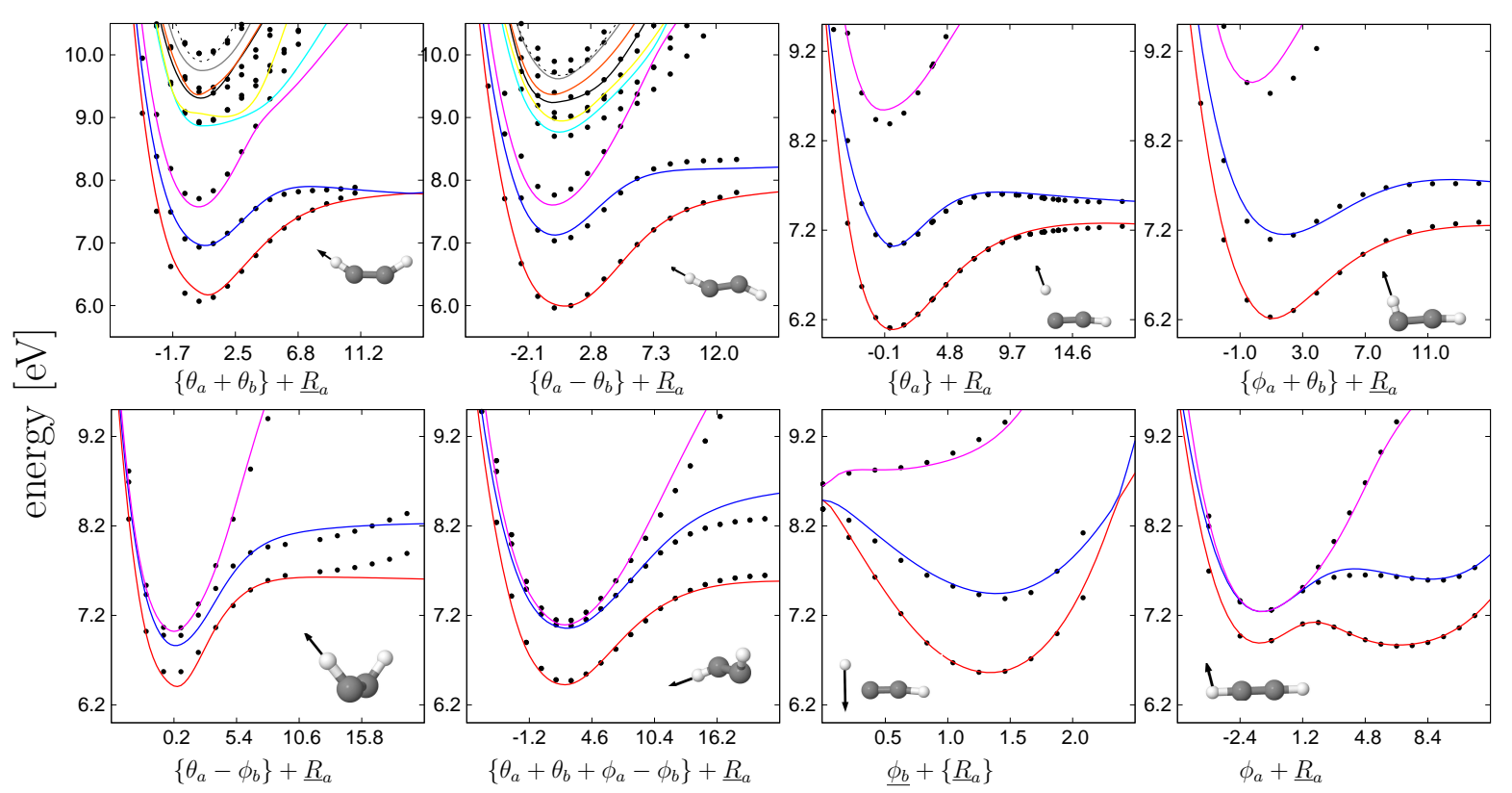

Fig. 6 Potential energy surface cuts of the fitted model with calculated energies correlating $\theta, \phi$ to $R_{a \mid b}$ coordinates for $\mathrm{S}_{1}-\mathrm{S}_{3}$ (and up to $\mathrm{S}_{9}$ for cis/trans dissociation, shown in top-left panels). Curly brackets mean the coordinate is kept constant but not at reference geometry. Energy in $\mathrm{eV}$, coordinates in $\AA[\mathrm{amu}]^{\frac{1}{2}}$ for $R$ or radians for angles. For those mixed angular/radial/normal vectors, coordinate underlined (below) indicates which one is shown in the $\mathrm{x}$-axis of plots. From left to right: first row : cis dissociation $\left(\left\{\theta_{a}+\theta_{b}\right\}+R_{a}\right)$, trans dissociation $\left(\left\{\theta_{a}-\theta_{b}\right\}+\underline{R_{a}}\right)$, bent dissociation $\left(\left\{\theta_{a}\right\}+\underline{R_{a}}\right), 2 \mathrm{D}$ bent-dissociation $\left(\left\{\phi_{a}+\theta_{a}\right\}+\underline{R_{a}}\right)$; second row: out-of-plane dissociation $\left(\left\{\theta_{a}+\phi_{b}\right\}+\underline{R_{a}}\right)$, skewed, out-of-plane dissociation $\left(\left\{\theta_{a}+\theta_{b}+\phi_{a}-\phi_{b}\right\}+\underline{R_{b}}\right)$, angle while CH stretched $\left(\underline{\phi_{b}}+\left\{R_{b}\right\}\right)$, simultaneous $\left(\phi_{a}+\underline{R_{a}}\right)$

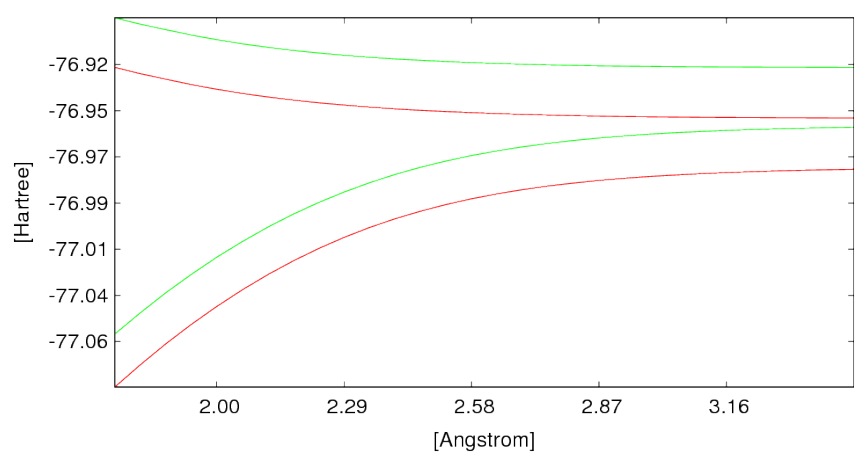

Fig. 7 Potential energy surfaces along the acetylene H-dissociation using CAS+RS2 (green) and MRCI+Davidson (red). No $\mathrm{S}_{0}-\mathrm{S}_{1}$ crossing was observed.

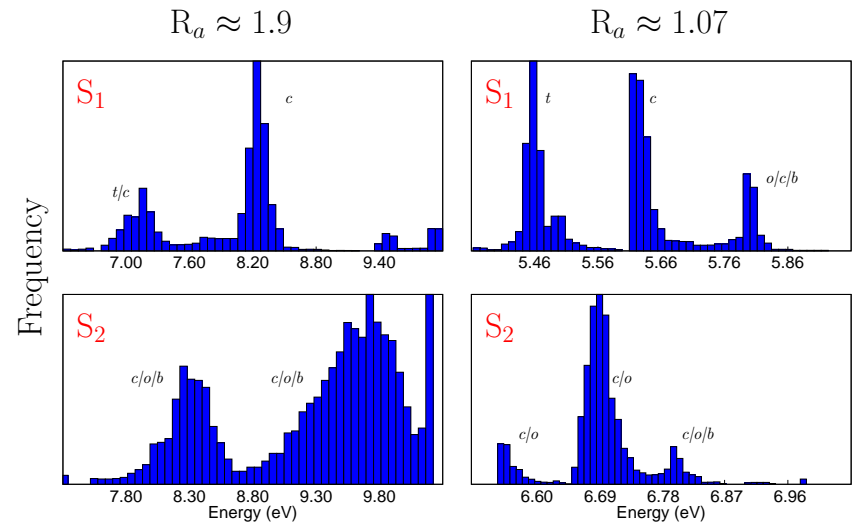

Fig. 8 Histogram of energy minima from optimisations started with initial geometries making a full DOF grid over the pertinent potential landscape. first row: left panel shows critical points of $S_{1}$ with one radial coordinate stretched to $1.9 \AA$, right $\mathrm{S}_{1}$ full optimisation. second row: left $\mathrm{S}_{2}$ as above right $\mathrm{S}_{2}$ as above. Peaks are labelled by the geometries that contribuited to them, with the following legend: $c$ : cis, $t$ : trans, $o$ : out-of-plane, $b$ : bent (weight contributed to the peak of geometry is written left-to-right). 
$\mathrm{S}_{2}$ (bottom left panel) the dissociation geometries favoured are out-of-plane or cisoid (cis like) and begin at $\sim 7.3 \mathrm{eV}$ with only small angles for one of the $\mathrm{CCH}$ angles (near 'bent' geometries), but increases significantly for the peak above $\sim 8.6$ eV.

\section{Dynamics}

Nuclear wave packet propagations were performed using the MCTDH algorithm ${ }^{38}$. The absorption spectra of a number of reduced dimensionality models were calculated, to serve as validation for the model. A number of propagations were then performed using the full-dimensional model to simulate VMD experiments.

\subsection{Numerical details}

Since the coordinates used are essentially a pair of spherical polar coordinates and a normal mode, the most natural choice of primitive basis function with which to construct the nuclear wavefunctions are the Legendre polynomials (being eigenfunctions of $L^{2}$ ), radial Harmonic Oscillator (HO) functions (for $R$ ) and harmonic oscillator eigen functions for the bound $Q_{c c s}$. The radial HO representation is defined on $[0, \infty)$ and has only the odd harmonic oscillator functions as a basis ${ }^{38}$, $\phi_{2 j-1}^{H O}(R)$, related to the Hermite polynomials. When diagonalizing the position operator, the weights of every discrete variable representation (DVR) grid point are given by:

$$
w_{\alpha}^{\frac{1}{2}} \propto R^{-1} \cdot U_{1 \alpha}
$$

where $\mathrm{U}$ is the matrix that transforms from the finite basis representation (FBR) to DVR representation. The factor $R^{-1}$ ensures the differential volume element from the Jacobian determinant ( $r^{2}$ factor) for the radial part of the spherical harmonic transformation is properly treated. This allows us to simplify the $\mathrm{KE}$ into the form given in equation 5. Mode combination for coordinates $\left\{\theta_{a}, \phi_{a}\right\}$ (and similarly for b) were used, since these are coupled via the kinetic energy operator (equation 5). The radial coordinates were also mode-combined, since they have many terms correlating them (Fig. 3).

\subsection{Calculated spectra}

A good test of the accuracy of a model Hamiltonian can be obtained by calculating its absorption spectra. The absorption spectra of acetylene is dominated by a long progression arising from $\mathrm{S}_{1}$ trans-bending mode as well as contributions arising from $\mathrm{CC}$ and possibly $\mathrm{CH}$ (totally symmetric) stretch modes $^{2,9,12}$. To confirm this, we calculated three different theoretical spectra from reduced dimensionality models; a 4-dimensional model of Renner-Teller, cis/trans coordinates $(\{\theta, \phi\})$, a 5-dimensional model including the $\mathrm{CC}$ symmetric stretch $\left(Q_{c c s}\right)$ and a full, 7-dimensional model further including the radial $\mathrm{CH}$ coordinates $\left(R_{a \mid b}\right)$. These are shown in Fig. 9.

A ground state wavefunction found by energy relaxation was initially operated on by the transition dipole from the $\left\langle S_{0}|\mu| S_{1}\right\rangle$ elements of this coordinate subspace using a linear approximation. By multiplying the autocorrelation of the resulting propagated wavefunction by a trigonometric damping function and Fourier transforming this product, we obtain a theoretical estimate for the experimental absorption spectra (Fig. 9). To reproduce such spectra, a nuclear wave-packet was propagated for $180 \mathrm{fs}$. For the angular coordinates, at least twelve single particle, two-dimensional functions (SPF) were used to represent the time dependent basis for each state, with sixteen for the first three excited states. In turn, the radial components required at least nine on each state with twelve on the first three excited states and the $\mathrm{CC}$ stretch required at least seven on each state.

The number of primitive grid points for $\theta$ and $\phi$ coordinates were 69 and 85 respectively. The $R$ coordinates required 131 in the range $[-8,55] \AA[\mathrm{amu}]^{\frac{1}{2}}$ and $Q_{c c s}$ required 79 in the range $[-20,55] \AA[\mathrm{amu}]^{\frac{1}{2}}$. The 4D model was the subject of an earlier article ${ }^{9}$, and it is also presented here to contrast the effect of the additional coordinates. Both the 5D and 7D spectra gave results that qualitatively match the experimental spectrum (Fig. 9) and resemble each other significantly, suggesting that the radial $\mathrm{CH}$ vibrations may not be significantly excited during the excitation, in accord with the fact that valence $\pi \pi^{*}$ orbital excitations typically don't significantly affect the $\mathrm{CH}$ bond length. This gives us some confidence the model reproduces qualitatively the excited state behaviour of acetylene.

\subsection{Modelling VMD}

The introduction describes how experimentalist have performed energy resolved VMD experiments probing the range 5.5-7.0 eV. These were done by preparing the initial ground state wavefunction to have quanta along local dissociation modes ${ }^{39}$ with an IR field followed by UV-excitations to the appropriate energy band. Though there exist methods ${ }^{38}$ in the MCTDH package to explore the eigenfunctions of a vibronic Hamiltonian, locating the appropriate mode combination eigenstates require the computation of a large number of eigenfunctions making such a method prohibitively time consuming. Instead, using a Morse Hamiltonian to describe the ground state $R_{a \mid b}$ coordinate:

$$
H=\frac{p^{2}}{2 m}+D\left(e^{-2 \alpha\left(R_{a \mid b}-r_{0}\right)}-2 e^{-\alpha\left(R_{a \mid b}-r_{0}\right)}\right.
$$



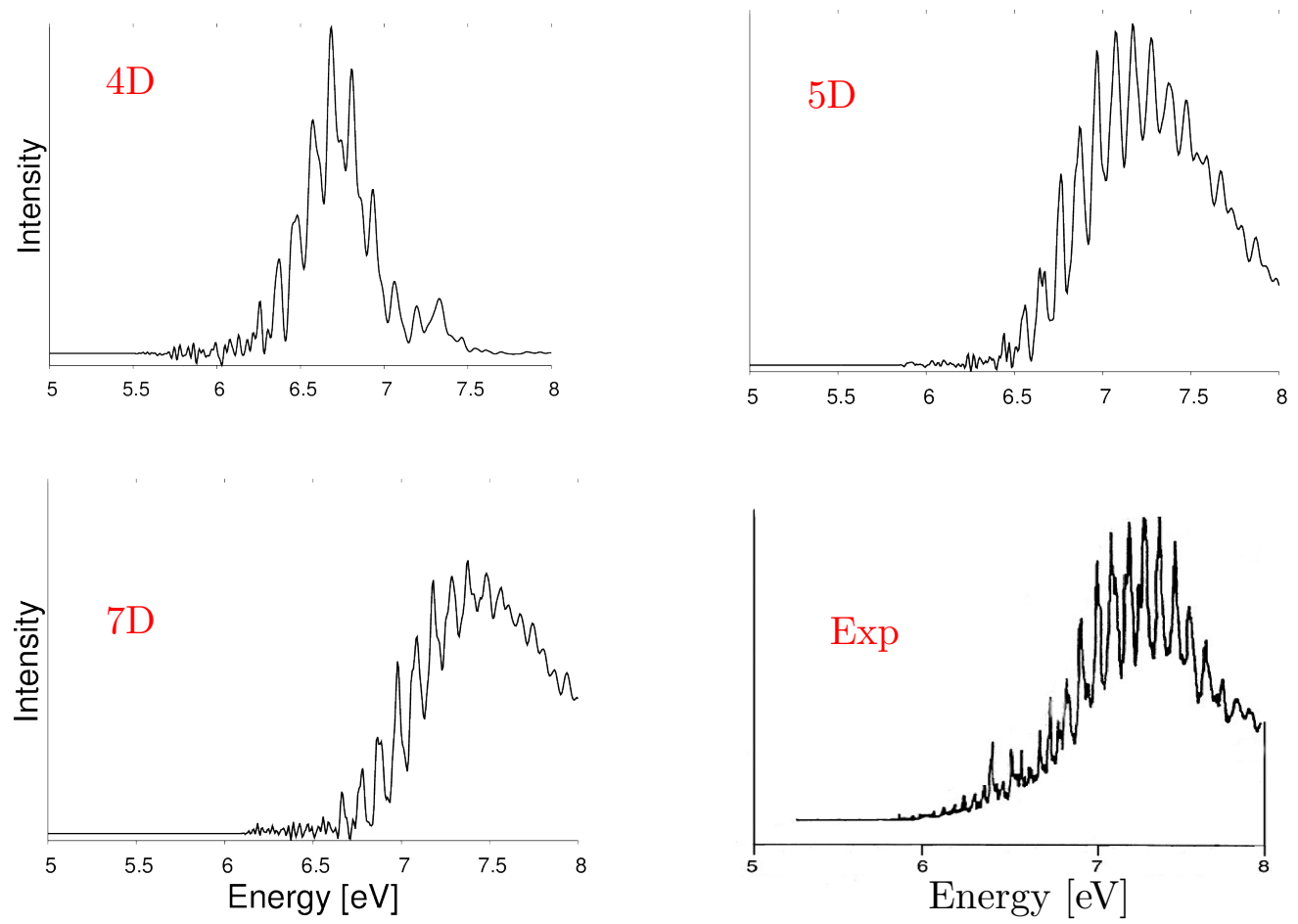

Fig. 9 top left: Calculated spectra for 4D Renner-Teller subspace, ten state model. top right: Calculated 5D spectra including CC stretch $\left(Q_{c c s}\right)$ in first excited state. bottom left: Calculated 7D spectra including radial coordinates $\left(R_{a \mid b}\right)$. bottom right: Experimental Spectra ${ }^{10}$; Image edited from Malsch et al ${ }^{12}$, providing assignments for cis/trans progressions in $\mathrm{S}_{1}-\mathrm{S}_{3}$.

initial wavefunctions were generated by applying an approximate raising operator on a previously relaxed ground state wavefunction 40 :

$$
\begin{aligned}
& L=\frac{1}{\sqrt{2 m \omega}}\left[\left(\Lambda-\frac{1}{2}\right) \alpha-\Lambda \alpha e^{-\alpha\left(\alpha\left(R_{a \mid b}-r_{0}\right)\right.}+i \cdot p\right] \\
& \omega=\alpha \sqrt{2 D / m} \quad \Lambda=\frac{\sqrt{2 D / m}}{\alpha}
\end{aligned}
$$

To simulate the UV-excitation in a narrow energy range, the laser field was modelled as a Gaussian envelope with the appropriate central frequency interacting with the transition dipole surface of the molecule. The transition dipole surfaces has been linearly approximated; Fig. 10 shows dipole surface cuts obtained from a CASSCF(6,9)//aug-cc-pVTZ wavefunction along the coordinates with significant contribution. These surfaces peak at trans geometries and cancel along cis for $\mathrm{S}_{1}$ and $\mathrm{S}_{2}$. The transition dipole along the z-component also has a significant value. To keep things manageable, in this work only excitation along the $\mathrm{x}$-component was considered. This should give identical results to using the y-component since the $\mathrm{x}$ and $\mathrm{y}$ vectors form $\mathrm{a} \Pi_{u}$ representation.

Like diabatic coupling, transition dipole coupling exhibit pJT like relations amongst the states. For example, from symmetry considerations, it is possible to show that $\left\langle\Sigma_{g}^{+}|\mu| \Delta_{u}\right\rangle$ transition dipole surfaces expanded to first order in nuclear

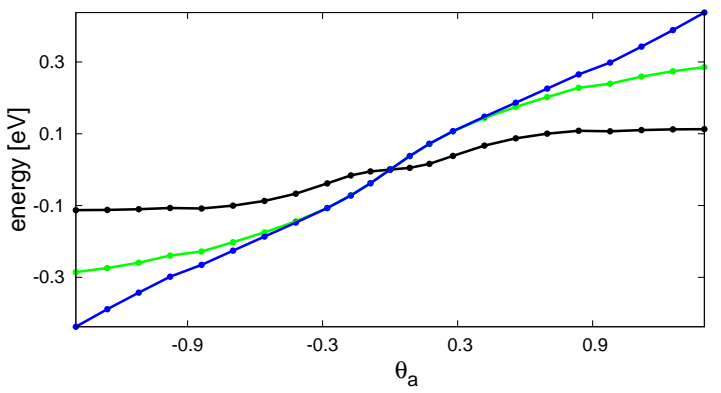

Fig. 10 Transition dipole along $\theta_{a}$; black: x-plane for $1 \Sigma_{u}^{-}$, blue: $\mathrm{x}$-plane for $1 \Delta_{u}^{x}$, green: $\mathrm{y}$-plane for $1 \Delta_{u}^{y}$. The sign of the surface is reversed for coordinate $\theta_{b}$.

coordinates have the following relations:

$$
\begin{aligned}
\left\langle\Sigma_{g}^{+}\left|\mu_{x}\left(\theta_{a}\right)\right| \Delta_{u}\right\rangle & =-\left\langle\Sigma_{g}^{+}\left|\mu_{y}\left(\theta_{b}\right)\right| \Delta_{u}\right\rangle=-\left\langle\Sigma_{g}^{+}\left|\mu_{x}\left(\theta_{b}\right)\right| \Delta_{u}\right\rangle= \\
\left\langle\Sigma_{g}^{+}\left|\mu_{y}\left(\theta_{a}\right)\right| \Delta_{u}\right\rangle & =+\left\langle\Sigma_{g}^{+}\left|\mu_{y}\left(\phi_{a}\right)\right| \Delta_{u}\right\rangle=-\left\langle\Sigma_{g}^{+}\left|\mu_{y}\left(\phi_{b}\right)\right| \Delta_{u}\right\rangle= \\
\left\langle\Sigma_{g}^{+}\left|\mu_{x}\left(\phi_{b}\right)\right| \Delta_{u}\right\rangle & =-\left\langle\Sigma_{g}^{+}\left|\mu_{x}\left(\phi_{a}\right)\right| \Delta_{u}\right\rangle
\end{aligned}
$$

Similar terms can be obtained for $\left\langle\Sigma_{g}^{+}|\mu| \Sigma_{u}^{-}\right\rangle$. The field was enveloped under a Gaussian function to avoid the cost of propagating a wavefunction under a permanent time-dependent external field; under such conditions, the constant mean-field integration scheme ${ }^{38}$, where the mean-field matrices are kept 
constant for longer time-steps than the single-particle functions and MCTDH coefficients, cannot be used. Due to computational limitations, it was only possible to model pulses in the range of tens of femtoseconds. Modelling a femtosecond laser pulse restricts the energy resolution that can be achieved as its Fourier decomposition will result in a spread of frequencies given by the uncertainty principle; a bandwidth with a full width at half maximum (FWHM) of $0.14 \mathrm{eV}$ was obtained with a pulse of $\Delta t=h / \Delta E=[\mathrm{eV} \cdot \mathrm{fs}] /[\mathrm{eV}]=4.135 / 0.14 \sim 30$ fs FWHM. The pulse is modelled using the following Gaussian envelope:

$$
\varepsilon(t)=\frac{s}{\sigma} \sqrt{\frac{4 \ln 2}{\pi}} \exp \left(-\frac{4 \ln 2}{\sigma^{2}}\left(t-t_{0}\right)^{2}\right) \cos \left(\omega\left(t-t_{0}\right)\right)
$$

where $\sigma$ is the FWHM, $s$ the strength parameter of the pulse and $\omega$ the central frequency.

As the excited vibrational states have resonances to different central frequencies, the population transferred is variable. However, to ensure that at least $1 \%$ of the population is transferred for all tests (in some instances we obtained full population transfer), the strength parameter was set to 70. Step functions were placed at the tails of the Gaussian envelope to help with numerics. After the pulse had ensued ( $\sim 65 \mathrm{fs}$ ), the propagation was halted and the CMF algorithm was used to continue the propagation for another 200 femtoseconds.

Six equidistant central frequencies with energies of 6.4-7.8 $\mathrm{eV}+\left(\mathrm{ZPE}_{S 0}\right)-\Delta^{I R}$ where used, where $\Delta^{I R}$ is the energy gained from the Morse raising operator. With this operator we 'IR' pre-excite wavefunctions with the fundamental and overtones 2, 3, 5 and 7 to test with the mentioned pulses. Complex absorbing potentials (CAP) were used to annihilate any components to the wavefunction that attempt to leave the grid along the $R_{a \mid b}$ coordinates. For the angular coordinates, at least nine two-dimensional SPF per state were required to ensure that the smallest SPF coefficient contributes $\sim 10^{-4}$ to the wavefunction. Similarly, at least six two-dimensional SPF per state were required for the radial coordinates and four for the $\mathrm{CC}$ stretch. With a sufficiently large grid we ensured the reciprocal grid in momentum space could describe the faster components of the wavefunction under the pulse field. This required 51 grid points for $\theta, 49$ for $\phi, 91$ for $R$ and 75 for $Q_{c c s}$.

Details for 36 propagations with varying initial overtone and central UV frequency are tabulated in 5. From this table, the following trends are apparent: with increasing total excitation energy more population is transferred from the ground state to excited states and greater dissociation results. Exciting with a total energy centred around $6.4 \mathrm{eV}$ might reduce the number of states available to populate (resonant), being near the first vibrational $S_{1}$ state. Although pre-exciting to higher overtones reduces the overall transfer of population when applying the UV-pulse, the flux across dissociation channels nevertheless increases significantly compared to no pre-excitation.

This is best illustrated, using the labelling in table 5 , by noting that propagation uv4-ir0 (no pre-excitation, but almost full transfer from the ground state) dissociates as much as uv4-ir5 (large pre-excitation, but less $0.5 \%$ transfer from the ground state). The model suggests that preparing the initial wavefunction in a higher local $\mathrm{CH}$ mode reduces the vibrational overlap between the ground state and electronic excited state vibrational wavefunctions but increases the energy imparted towards dissociation. We remind the reader that in these propagations the light pulse excites along trans vibrational modes, since these have the strongest transition dipole surface elements. However, since the transition dipole along $R$ are not negligible, it may be that this model does not accurately reproduce the correct transition vibrational overlap along these coordinates.

Fig. 11 shows the flux into the dissociation channel for all states over the $250 \mathrm{fs}$, for all 36 propagations. One can see that for pre-excited pulses most of the transfer occurs shortly after the pulse, whereas for the relaxed ground state wavefunction the density transferred often continues to accelerate across the dissociation region even after 200 fs.

The favoured geometries were determined at the time of dissociation during the first 265 fs of dynamics. Figs. 1321 provided as supplementary information (some examples reprinted in Fig. 12) show sets of frames of flux into the dissociating channels of $R_{a}$ within the coordinates $\phi_{a}$ (y-axis: $(\pi>$ $\left.\phi_{a}>\frac{\pi}{2}\right)$ ) and $\theta_{a}$ (x-axis: $\left(\pi>\theta_{a}>0\right)$ while keeping $\theta_{b}$ fixed in each frame $\left(\frac{\pi}{2}>\theta_{b}>0\right)$. Each frame shows a value of $\mathrm{i}$ for $\theta_{b}=\left(\pi / 2-\frac{\pi / 2 \times i}{10}\right), i=0,1, \ldots, 9$. Note that $\theta_{b}=\pi / 2$ (in equilibrium). $\phi_{a}$ roughly describes out-of-plane geometries (when $\theta_{a}$ and $\theta_{b} \neq$ equilibrium), while $\theta_{a}<\frac{\pi}{2}$ corresponds to trans conformations and $\theta_{a}>\frac{\pi}{2}$ to $\operatorname{cis}$ (for $\theta_{b} \neq \frac{\pi}{2}$, away from the equilibrium geometry). Throughout all maps $\phi_{b}=\pi$, fixed in the equilibrium geometry. In other words, each set of frames shows a 3D subspace representing distinguishable geometries (i.e. trans, cis, linear, bent and out-of-plane geometries), with a colour map determining the flux across that dissociation channel. By bent geometry we mean one of the bond angles is $180^{\circ}$.

It should be mentioned that in the present experiment, where we modelled a plane polarised pulse along the xz-plane, the flux across trans, cis motion in the yz-plane should not result in the same as the xz-plane (therefore not shown in figures). Each frame was obtained by generating a collection of geometry grid points representing the final flux for $R_{a}$ dissociation of the wavefunction around this region. The flux in this subspace is obtained by first acting on the wavefunction with a product of box functions so as to only leave density around the 


\begin{tabular}{|c|c|c|c|c|c|c|c|}
\hline Label & $\begin{array}{l}\text { Tot En } \\
(\mathrm{eV})^{a}\end{array}$ & $\mathrm{MO}^{b}$ & $\begin{array}{l}\text { GS En } \\
(\mathrm{eV})^{c}\end{array}$ & $\begin{array}{l}\text { UV En } \\
(\mathrm{eV})^{d}\end{array}$ & $\% S_{0}$ Pop $^{e}$ & $\begin{array}{c}\text { Tot } \\
\text { Diss }^{f}\end{array}$ & $\begin{array}{c}\text { Tot } \\
\text { Diss }^{g}\end{array}$ \\
\hline uv0-ir0 & & $\overline{0}$ & 0.76 & 5.64 & 0.93272611 & 0.00001 & 0.01035 \\
\hline uv0-ir1 & & 1 & 1.24 & 5.16 & 0.99513311 & 0.00001 & 0.00219 \\
\hline uv0-ir2 & 640 & 2 & 1.45 & 4.95 & 0.99889976 & 0.00003 & 0.00216 \\
\hline uv0-ir3 & 0.40 & 3 & 1.69 & 4.71 & 0.99984823 & 0.00006 & 0.00208 \\
\hline uv0-ir4 & & 5 & 2.21 & 4.19 & 0.99994895 & 0.00166 & 0.00826 \\
\hline uv0-ir5 & & 7 & 2.73 & 3.67 & 0.99983620 & 0.03261 & 0.08193 \\
\hline uv1-ir0 & & 0 & 0.76 & 5.92 & 0.80290768 & 0.00002 & 0.03805 \\
\hline uv1-ir1 & & 1 & 1.24 & 5.44 & 0.97325940 & 0.00004 & 0.00853 \\
\hline uv1-ir2 & & 2 & 1.45 & 5.23 & 0.99135512 & 0.00016 & 0.02151 \\
\hline uv1-ir3 & 6.68 & 3 & 1.69 & 4.99 & 0.99791293 & 0.00045 & 0.02577 \\
\hline uv1-ir4 & & 5 & 2.21 & 4.47 & 0.99977558 & 0.00170 & 0.01856 \\
\hline uv1-ir5 & & 7 & 2.73 & 3.95 & 0.99937751 & 0.00184 & 0.05784 \\
\hline uv2-ir0 & & 0 & 0.76 & 6.20 & 0.63214908 & 0.00011 & 0.14830 \\
\hline uv2-ir1 & & 1 & 1.24 & 5.72 & 0.90362589 & 0.00050 & 0.03097 \\
\hline uv2-ir2 & & 2 & 1.45 & 5.51 & 0.96092724 & 0.00203 & 0.09330 \\
\hline uv2-ir3 & 6.96 & 3 & 1.69 & 5.27 & 0.98791055 & 0.00902 & 0.16378 \\
\hline uv2-ir4 & & 5 & 2.21 & 4.75 & 0.99849079 & 0.02113 & 0.11037 \\
\hline uv2-ir5 & & 7 & 2.73 & 4.23 & 0.99840603 & 0.10555 & 0.46281 \\
\hline uv3-ir0 & & 0 & 0.76 & 6.48 & 0.44770916 & 0.00034 & 0.46996 \\
\hline uv3-ir1 & & 1 & 1.24 & 6.00 & 0.75210196 & 0.00156 & 0.16474 \\
\hline uv3-ir2 & 70 & 2 & 1.45 & 5.79 & 0.86979412 & 0.00807 & 0.81784 \\
\hline uv3-ir3 & 7.24 & 3 & 1.69 & 5.55 & 0.94852116 & 0.04258 & 1.07881 \\
\hline uv3-ir4 & & 5 & 2.21 & 5.03 & 0.99267406 & 0.34027 & 1.13715 \\
\hline uv3-ir5 & & 7 & 2.73 & 4.51 & 0.99669197 & 0.96607 & 2.54839 \\
\hline uv4-ir0 & & $\overline{0}$ & 0.76 & 6.76 & 0.10565797 & 0.00224 & 2.17146 \\
\hline uv4-ir1 & & 1 & 1.24 & 6.28 & 0.59616333 & 0.00616 & 0.30913 \\
\hline uv4-ir2 & 7 & 2 & 1.45 & 6.07 & 0.73817475 & 0.01522 & 1.01600 \\
\hline uv4-ir3 & 1.52 & 3 & 1.69 & 5.83 & 0.84498736 & 0.12002 & 4.61980 \\
\hline uv4-ir4 & & 5 & 2.21 & 5.31 & 0.97359007 & 0.67901 & 3.47766 \\
\hline uv4-ir5 & & 7 & 2.73 & 4.79 & 0.99154697 & 2.67559 & 6.33137 \\
\hline uv5-ir0 & & 0 & 0.76 & 7.04 & 0.04684333 & 0.00636 & 8.57936 \\
\hline uv5-ir1 & & 1 & 1.24 & 6.56 & 0.37867083 & 0.01286 & 0.98093 \\
\hline uv5-ir2 & & 2 & 1.45 & 6.35 & 0.57932659 & 0.04972 & 1.72780 \\
\hline uv5-ir3 & 7.80 & 3 & 1.69 & 6.11 & 0.69098010 & 0.15060 & 11.9383 \\
\hline uv5-ir4 & & 5 & 2.21 & 5.59 & 0.92338604 & 0.41226 & 8.03986 \\
\hline uv5-ir5 & & 7 & 2.73 & 5.07 & 0.97904797 & 0.95607 & 4.33664 \\
\hline
\end{tabular}

Table 5 Results from a series of propagations of acetylene with different initial IR + UV excitations. $a$ Total energy (MO excitation + central pulse) includes Zero Point Energy. ; $b$ M.O. = Morse potential Overtone. ; $c$ Ground state energy after using Morse ladder Operator (includes ZPE). ; $d$ Central Frequency of pulse. ; $e$ Population remaining in ground state after pulse. ; $f=$ Density (x1000) absorbed by dissociation CAP's after Pulse (65fs). ; $g$ = Final density (x1000) absorbed by dissociation CAP's after Pulse (265 fs). 

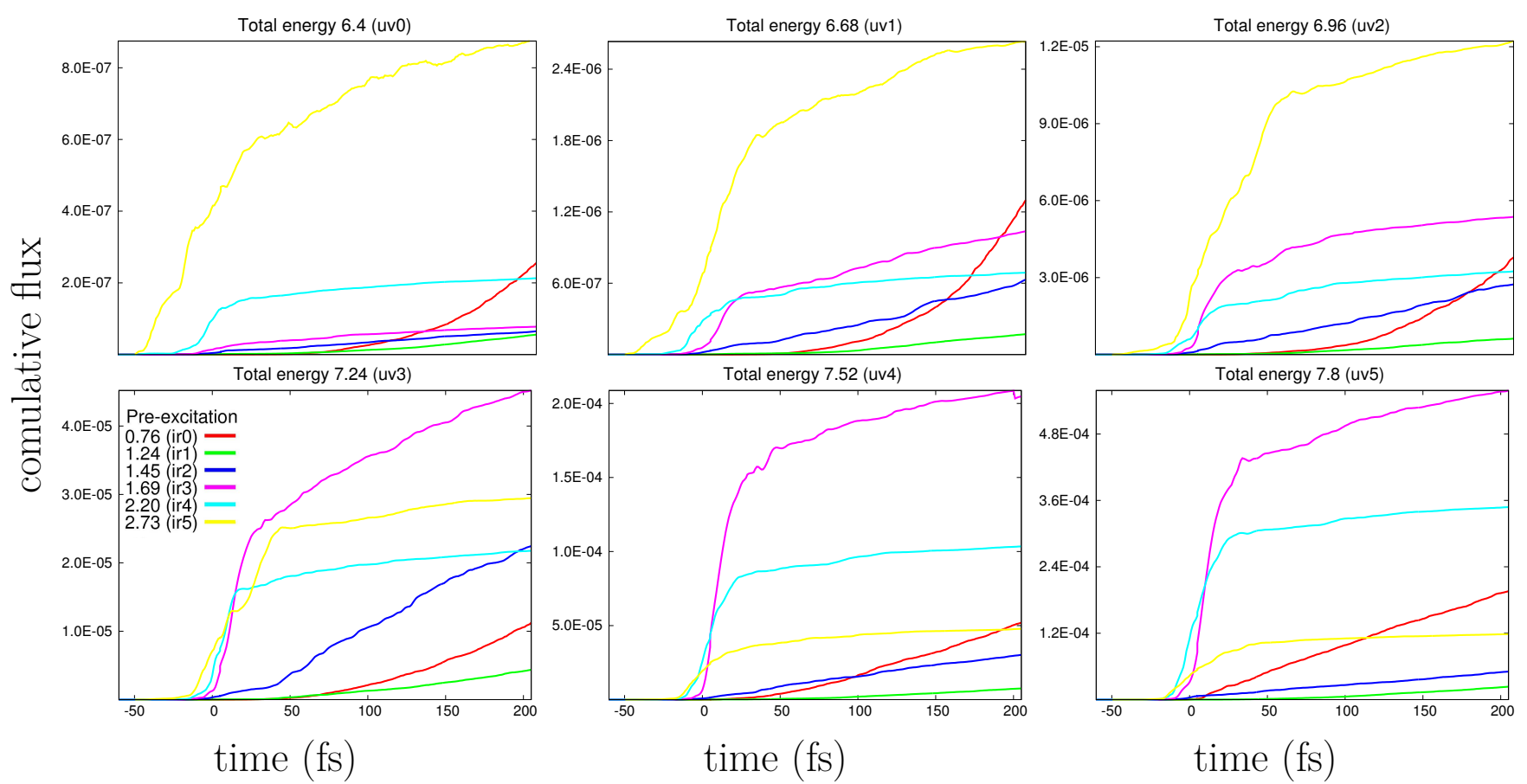

Fig. 11 Cumulative flux into the dissociation channel along $R_{a}$ for 36 simulations. Time $=0$ fs determines when the UV-pulse has finished. Labels for propagations refer to Tab 5 and to flux maps in Supplementary information.
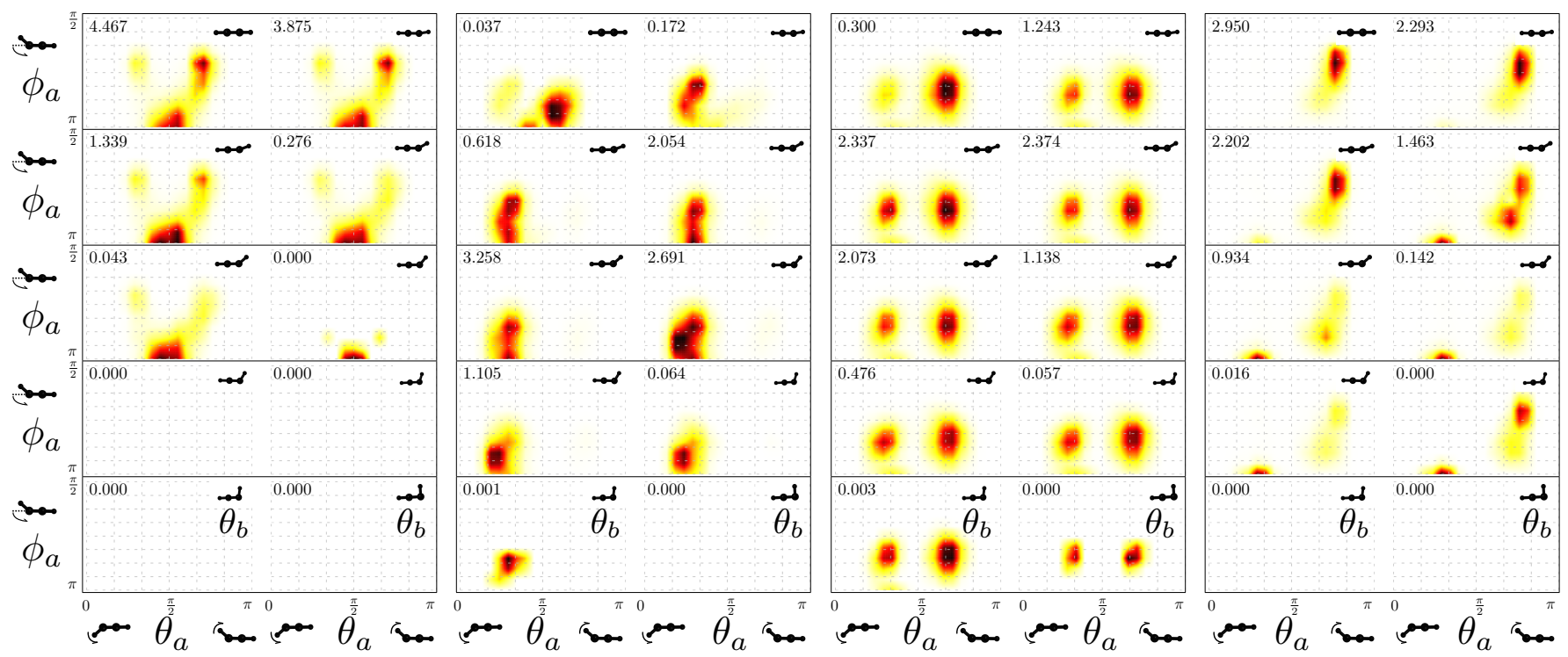

Fig. 12 Four representative panels displaying the total flux for all states into the $R_{a}$ dissociation channel for 200 fs after the UV-pulse has ended, within a 3D, angular-coordinate subspace (in ten 2D frames). Coordinate dimensions in radians. The y-axis correspond to $\phi_{a}$ the azimuthal angle: $\pi$ corresponds to the $\mathrm{CCH}$ linear conformation (bottom of the y-axis) and at $\frac{\pi}{2}$ (the top of the frame) corresponds to an out-of-plane geometry (with respect to atom $b$ ). The x-axis corresponds to the $\theta_{a}$ polar angle: $\frac{\pi}{2}$ corresponds to the $\mathrm{CCH}$ linear geometry (and located half-way along the $\mathrm{x}$-axis) and with respect to atom $b$ it forms a trans (left side of $\mathrm{x}$-axis) or cis (right side of $\mathrm{x}$-axis). Each frame corresponds to a value of the polar angle of atom $b, 0<\theta_{b}<\frac{\pi}{2}$. Each frame has a number in the top LHS of the frame which provides the contribution each frame gives to the total flux into the $R_{a}$ dissociation channel (normalised to 10.0). In brackets (Total E (eV), Morse Overtone). from left to right : uv4-ir0 (7.52,0) - near linear ; uv1-ir2 (6.68,2) - trans (with slight out of plane) ; uv0-ir4 (6.4 ,5) - mixture of cis and trans (with slight out of plane) ; uv4-ir4 (7.52,5) - bent and cis (with strong out of plane) 
specific grid geometry $\theta_{a}=\theta_{a 0}, \phi_{a}=\phi_{a 0}, \theta_{b}=\theta_{b 0}, \phi_{b}=\pi$ :

$$
\begin{gathered}
\hat{\Theta}|\Psi\rangle= \\
{\left[\left[\Theta^{+}\left(\theta_{a}-\theta_{a 0}^{+}\right) \cdot \Theta^{-}\left(\theta_{a}-\theta_{a 0}^{-}\right)\right] .\right.} \\
{\left[\Theta^{+}\left(\phi_{a}-\phi_{a 0}^{+}\right) \cdot \Theta^{-}\left(\phi_{a}-\phi_{a 0}^{-}\right)\right] \cdot} \\
{\left[\Theta^{+}\left(\theta_{b}-\theta_{b 0}^{+}\right) \cdot \Theta^{-}\left(\theta_{b}-\theta_{b 0}^{-}\right)\right] \cdot} \\
\left.\left[\Theta^{+}\left(\phi_{b}-\frac{21 \pi}{20}\right) \cdot \Theta^{-}\left(\phi_{b}-\frac{19 \pi}{20}\right)\right]\right]|\Psi\rangle
\end{gathered}
$$

where $\Theta^{ \pm}$are the forward and backward step functions. $\theta_{a 0}^{ \pm}=$ $\theta_{a 0} \pm \frac{\pi}{20}$ Thus by setting $\theta_{a 0}^{ \pm}$so as to make a $4 \mathrm{D}$ box function of width $\frac{\pi}{10}$ and centred at $\left\{\theta_{a}=\theta_{a 0}, \phi_{a}=\phi_{a 0}, \theta_{b}=\theta_{b 0}\right\}$. The flux is evaluated by taking the expectation value of the commutator of a step function along $R_{a}$ with the kinetic energy operator:

$$
\left\langle\Psi\left|\left[\Theta^{+}\left(R_{a}-R_{\text {diss }}\right), \hat{p}_{i}^{2}+\frac{\hat{L}_{i}^{2}}{R_{a}^{2}}\right]\right| \Psi\right\rangle
$$

Table 6 clarifies the results by giving the most likely geometries at the moment of dissociation for a given IR preexcitation (Morse overtone using the ladder operator) and UV pulse. The observations and predictions this model makes can be summarised as follows:

- Exciting in the region of 6-7 eV (including ZPE) should lead to dissociation in a slightly out-of-plane trans geometry, unless pre-excited with more than $2.2 \mathrm{eV}$ along the $\mathrm{CH}$ vibration, where the molecule is likely to dissociate at near-linear and bent geometries. These channels are likely to belong to $S_{1}$.

- Exciting above $7 \mathrm{eV}$ (including ZPE from the ground state a mixture of out-of-plane cis and trans geometries are obtained. These channels are likely to belong to $S_{1}$.

- Strong out-of-plane, cis geometries are obtained by exciting from high overtones to energies in the 7.5-7.8 eV range. These channels are likely to belong to $S_{2}$.

- No pure cis dissociating geometries were seen, having at least some out-of-plane contribution. No cis was found below $\sim 7 \mathrm{eV}$

Since we are working in a diabatic representation which was built from strongly mixed adiabatic states and a pulse which results in an energy band of $0.14 \mathrm{eV}$ width, it was not possible

\begin{tabular}{|c|c|c|c|c|}
\hline \multirow[b]{2}{*}{$\begin{array}{l}\text { Over } \\
\text { tone }\end{array}$} & \multicolumn{4}{|c|}{ Total Energy (eV): IR + UV } \\
\hline & $\begin{array}{ccc}6.4 & 6.68 & 6.96 \\
\text { (uv0) } & \text { (uv1) } & \text { (uv2) }\end{array}$ & $\begin{array}{l}7.24 \\
\text { (uv3) }\end{array}$ & $\begin{array}{l}7.52 \\
(u v 4)\end{array}$ & $\begin{array}{c}7.8 \\
\text { (uv5) }\end{array}$ \\
\hline $\begin{array}{c}0 \\
\text { (ir0) }\end{array}$ & [T2-P1] [T2-P1] [T2-P1] & T2-P1] & $\begin{array}{l}{[\mathrm{C} 2-\mathrm{P} 2]} \\
{[\mathrm{T} 2-\mathrm{P} 2]}\end{array}$ & $\begin{array}{l}{[\mathrm{B} 2-\mathrm{P} 2]} \\
{[\mathrm{T} 2-\mathrm{P} 2]} \\
{[\mathrm{C} 2-\mathrm{P} 2]}\end{array}$ \\
\hline $\begin{array}{c}1 \\
\text { (ir1) }\end{array}$ & [T2-P1] [T2-P1] [T2-P1] & [T2-P2] & [T2-P2] & [T2-P2] \\
\hline$\stackrel{2}{2}$ & {$[\mathrm{~T} 2-\mathrm{P} 0][\mathrm{T} 2-\mathrm{P} 0]$ [T2-P0] } & [T2-P1] & [T2-P0] & [T1-P0] \\
\hline $\begin{array}{c}3 \\
\text { (ir3) }\end{array}$ & $\left.\begin{array}{l}{[\mathrm{L}-\mathrm{P} 0]} \\
{[\mathrm{B} 2-\mathrm{P} 2]}\end{array}\right]$ T2-P0] [T2-P0] & $2-\mathrm{P}$ & 2-P0 & T1-P0] \\
\hline $\begin{array}{c}5 \\
\text { (ir4) }\end{array}$ & $\begin{array}{l}{[\mathrm{L}-\mathrm{P} 0][\mathrm{L}-\mathrm{P} 0][\mathrm{L}-\mathrm{P} 0]} \\
{[\mathrm{B} 2-\mathrm{P} 1][\mathrm{B} 2-\mathrm{P} 2][\mathrm{B} 2-\mathrm{P} 2]}\end{array}$ & {$[\mathrm{B} 2-\mathrm{P} 2$} & $\begin{array}{l}{[\mathrm{B} 2-\mathrm{P} 3]} \\
{[\mathrm{C} 2-\mathrm{P} 2]} \\
\end{array}$ & $\begin{array}{l}{[\mathrm{B} 2-\mathrm{P} 3]} \\
{[\mathrm{C} 2-\mathrm{P} 3]} \\
\end{array}$ \\
\hline $\begin{array}{c}7 \\
\text { (ir5) }\end{array}$ & {$[\mathrm{L}-\mathrm{P} 0][\mathrm{L}-\mathrm{P} 0][\mathrm{L}-\mathrm{P} 0]$} & $\begin{array}{l}{[\mathrm{B} 2-\mathrm{P}} \\
{[\mathrm{T} 2-\mathrm{P}} \\
{[\mathrm{C} 2-\mathrm{P}} \\
\end{array}$ & $\begin{array}{l}{[\mathrm{B} 2-\mathrm{P} 3} \\
{[\mathrm{C} 2-\mathrm{P} 2}\end{array}$ & $\begin{array}{l}{[\mathrm{B} 2-\mathrm{P} 3]} \\
{[\mathrm{C} 2-\mathrm{P} 3]} \\
\end{array}$ \\
\hline
\end{tabular}
to determine with confidence to which adiabatic states these different dissociating channels belong to. The adiabatic dissociation channels suggested in the above summary are based on the minima shown in Fig. 8, basins suggested by Fig. 8 and geometries in Tab 1.
Table 6 Approximate geometries of dissociating $\mathrm{H}$ atom based on VMD flux maps like those in Fig. 12. Legend:

letters: $\mathrm{T}=$ trans, $\mathrm{C}=$ cis, $\mathrm{B}=$ bent, $\mathrm{L}=$ linear, $\mathrm{P}=$ out-of-plane coordinate contribution to T or C. numbers: $0=0^{\circ}, 1=\frac{\pi}{8}^{\circ}, 2=\frac{2 \pi}{8}^{\circ}, 3=\frac{3 \pi}{8}^{\circ}$, radians away from equilibrium geometries referring to $\mathrm{T}, \mathrm{C}, \mathrm{B}$ or $\mathrm{P}$. By bent geometry we mean one of the bond angles is $180^{\circ}$. Note that for B geometries P cannot refer to out-of plane geometries (dihedral angle not defined), but continues to be a bent molecule with $\mathrm{P}$ only describing the location of the density in the 2D flux maps (corresponding to a rotation about the z-axis of the bent molecule). 


\section{Conclusions}

The $\Sigma_{g}^{+}$symmetry adapted polynomials basis generated elsewhere $^{9}$ were used to fit a full internal DOF, 10 state model of acetylene. The model includes Renner-Teller and pseudo Jahn Teller coupling along dissociation coordinates. To use this basis, coordinates forming a basis for $\mathrm{D}_{\infty h}$ irreducible representations were devised by a spherical polar transformation from rotated normal coordinates. Ten states were required to properly fit the first three excited states using these 1D product-form functions. The model agrees well with the critical points reported in the literature, correctly describes the $S_{1}$ and $S_{2 / 3}$ minima as well as out of plane geometries The calculated experimental absorption spectra agrees qualitatively with the experimental one. This served as a validation for the model which was then used to simulate VMD experiments akin to those found in the literature. This was done by operating the initial ground state wavefunction with Morse ladder operators, mimicking IR pre-excitation, followed by a Gaussian enveloped sinusoidal function representing a experimental pulse polarised laser field. An increase in dissociation yield was obtained by increasing pre-excitation, despite the decrease in population-transfer away from the ground state, owing to a reduced vibrational overlap with excited states. The model suggests that for energies in the range 6.4-7.0 eV, $\mathrm{S}_{1}$ can give trans or linear/bent geometries depending on the IR pre-excitation of $\mathrm{CH}$ stretch local modes. Similar control can be obtained in the range $7-8 \mathrm{eV}$ for state $S_{2}$, where we can also obtain dissociation at $c$ is geometries. These results complement and agree with many of the observations reported in the literature; Indeed the trans mode plays an important part in the dissociation mechanism, this work adds further light into the dynamic behaviour of this fundamental system.

\section{References}

1 R. P. Schmid, T. ArusiParpar, R. J. Li, I. Bar and S. Rosenwaks, Journal of Chemical Physics, 1997, 107, 385-391.

2 C. K. Ingold and G. W. King, Journal of the Chemical Society, 1953, 2702-2704.

3 J. K. Lundberg, Y. Q. Chen, J. P. Pique and R. W. Field, Journal of Molecular Spectroscopy, 1992, 156, 104-122.

4 Y. L. Yung, M. Allen and J. P. Pinto, Astrophysical Journal Supplement Series, 1984, 55, 465-506.

5 T. R. Fletcher and S. R. Leone, Journal of Chemical Physics, 1989, 90, 871-879.

6 B. Lasorne, G. A. Worth and M. A. Robb, in Molecular Quantum Dynamics, ed. F. Gatti, Springer, Heidelberg, Germany, 2014, pp. 181-211.

7 H. Köppel, W. Domcke and L. S. Cederbaum, Adv. Chem. Phys., 1984, 57, 59-246.

8 G. A. Worth, H.-D. Meyer, H. Köppel, L. S. Cederbaum and I. Burghardt, Int. Rev. Phys. Chem., 2008, 27, 569-606.

9 C. Robertson and G. A. Worth, Chemical Physics, 2015, 460, 125-134.

10 T. Nakayama and K. Watanabe, Journal of Chemical Physics, 1964, 40, 558 -
11 F. Laruelle, S. Boye-Peronne, D. Gauyacq and J. Lievin, Journal of Physical Chemistry A, 2009, 113, 13210-13220.

12 K. Malsch, G. Hohlneicher, R. Schork and H. Koppel, Physical Chemistry Chemical Physics, 2001, 3, 5393-5407.

13 Q. Cui and K. Morokuma, Chemical Physics Letters, 1997, 272, 319-327.

14 L. S. Cederbaum, H. Koppel and W. Domcke, International Journal of Quantum Chemistry, 1981, 251-267.

15 Y. Liu, I. B. Bersuker, W. Zou and J. E. Boggs, Chemical Physics, 2010, 376, 30-35.

16 P. Garcia-Fernandez and I. B. Bersuker, International Journal of Quantum Chemistry, 2012, 112, 3025-3032.

17 A. Haijima, M. Fujii and M. Ito, Journal of Chemical Physics, 1990, 92, 959-968.

18 Q. Cui, K. Morokuma and J. F. Stanton, Chemical Physics Letters, 1996, 263, 46-53.

19 P. Dupre, R. Jost, M. Lombardi, P. G. Green, E. Abramson and R. W. Field, Chemical Physics, 1991, 152, 293-318.

20 M. Fujii, S. Tanabe, Y. Okuzawa and M. Ito, Laser Chemistry, 1994, 14, 161-182.

21 Y. Ganot, A. Golan, X. Z. Sheng, S. Rosenwaks and I. Bar, Physical Chemistry Chemical Physics, 2003, 5, 5399-5404.

22 E. Ventura, M. Dallos and H. Lischka, Journal of Chemical Physics, 2003, 118, 1702-1713.

23 S. J. Humphrey, C. G. Morgan, A. M. Wodtke, K. L. Cunningham, S. Drucker and R. W. Field, Journal of Chemical Physics, 1997, 107, 49-53.

24 K. L. Bittinger, W. L. Virgo and R. W. Field, Journal of Physical Chemistry A, 2011, 115, 11921-11943.

25 D. H. Mordaunt, M. N. R. Ashfold, R. N. Dixon, P. Loffler, L. Schnieder and K. H. Welge, Journal of Chemical Physics, 1998, 108, 519-526.

26 T. M. Ticich, M. D. Likar, H. R. Dubal, L. J. Butler and F. F. Crim, Journal of Chemical Physics, 1987, 87, 5820-5829.

27 B. Schubert, H. Koppel and H. Lischka, Journal of Chemical Physics, 2005, 122, 184312.

28 M. P. Irion and K. L. Kompa, Applied Physics B-Photophysics and Laser Chemistry, 1982, 27, 183-186.

29 A. M. Wodtke and Y. T. Lee, Journal of Physical Chemistry, 1985, 89, 4744-4751.

30 R. P. Schmid, Y. Ganot, T. Arusi-Parpar, R. J. Li, I. Bar and S. Rosenwaks, in State-selective dissociation of acetylene isotopomers, ed. J. W. Hepburn, R. E. Continetti and M. A. Johnson, 1998, vol. 3271, pp. 28-35.

31 Y. Ganot, X. Z. Sheng, I. Bar and S. Rosenwaks, Chemical Physics Letters, 2002, 361, 175-181.

32 M. J. Frisch, G. W. Trucks, H. B. Schlegel, G. E. Scuseria, M. A. Robb, J. R. Cheeseman, J. A. Montgomery, Jr., T. Vreven, K. N. Kudin, J. C. Burant, J. M. Millam, S. S. Iyengar, J. Tomasi, V. Barone, B. Mennucci, M. Cossi, G. Scalmani, N. Rega, G. A. Petersson, H. Nakatsuji, M. Hada, M. Ehara, K. Toyota, R. Fukuda, J. Hasegawa, M. Ishida, T. Nakajima, Y. Honda, O. Kitao, H. Nakai, M. Klene, X. Li, J. E. Knox, H. P. Hratchian, J. B. Cross, V. Bakken, C. Adamo, J. Jaramillo, R. Gomperts, R. E. Stratmann, O. Yazyev, A. J. Austin, R. Cammi, C. Pomelli, J. W. Ochterski, P. Y. Ayala, K. Morokuma, G. A. Voth, P. Salvador, J. J. Dannenberg, V. G. Zakrzewski, S. Dapprich, A. D. Daniels, M. C. Strain, O. Farkas, D. K. Malick, A. D. Rabuck, K. Raghavachari, J. B. Foresman, J. V. Ortiz, Q. Cui, A. G. Baboul, S. Clifford, J. Cioslowski, B. B. Stefanov, G. Liu, A. Liashenko, P. Piskorz, I. Komaromi, R. L. Martin, D. J. Fox, T. Keith, M. A. Al-Laham, C. Y. Peng, A. Nanayakkara, M. Challacombe, P. M. W. Gill, B. Johnson, W. Chen, M. W. Wong, C. Gonzalez and J. A. Pople, Gaussian 03, Revision C.02.

33 H.-J. Werner, P. J. Knowles, G. Knizia, F. R. Manby, M. Schütz, P. Celani, T. Korona, R. Lindh, A. Mitrushenkov, G. Rauhut, K. R. Shamasundar, T. B. Adler, R. D. Amos, A. Bernhardsson, A. Berning, D. L. Cooper, M. J. O. Deegan, A. J. Dobbyn, F. Eckert, E. Goll, C. Hampel, A. Hes- 
selmann, G. Hetzer, T. Hrenar, G. Jansen, C. Köppl, Y. Liu, A. W. Lloyd, R. A. Mata, A. J. May, S. J. McNicholas, W. Meyer, M. E. Mura, A. Nicklass, D. P. O'Neill, P. Palmieri, D. Peng, K. Pflüger, R. Pitzer, M. Reiher, T. Shiozaki, H. Stoll, A. J. Stone, R. Tarroni, T. Thorsteinsson and M. Wang, MOLPRO, version 2012.1, a package of ab initio programs, 2012.

34 L. Liu and J. T. Muckerman, Journal of Chemical Physics, 1997, 107, 3402-3416.

35 N. C. Handy, Molecular Physics, 1987, 61, 207-223.

36 F. S. Crawford, American Journal of Physics, 1964, 32, 611-.

37 C. Robertson and G. A. Worth, to be published.

38 M. H. Beck, A. Jackle, G. A. Worth and H. D. Meyer, Physics ReportsReview Section of Physics Letters, 2000, 324, 1-105.

39 J. Zhang, C. W. Riehn, M. Dulligan and C. Wittig, Journal of Chemical Physics, 1995, 103, 6815-6818.

40 G. A. Worth, M. H. Beck, A. Jäckle and H.-D. Meyer, The Heidelberg MCTDH Package: A set of programs for multi-dimensional quantum dynamics. User's Guide, Version 8, 2000

(The User's Guide can be downloded from the URL: http://www.pci.uniheidelberg.de/tc/usr/mctdh/). 